Mathematical Modelling and Analysis

Volume 20 Number 2, March 2015, 205-231

http://dx.doi.org/10.3846/13926292.2015.1020894

(c) Vilnius Gediminas Technical University, 2015
Publisher: Taylor\&Francis and VGTU

http://www.tandfonline.com/TMMA

ISSN: $1392-6292$

eISSN: $1648-3510$

\title{
Global Solution for the Generalized Anisotropic Navier-Stokes Equations with Large Data
}

\section{Daoyuan Fang ${ }^{a}$ and Bin Han $^{a, b}$}

${ }^{a}$ Department of Mathematics, Zhejiang University

Hangzhou, China

${ }^{b}$ Department of Mathematics, Fudan University

Shanghai, China

E-mail(corresp.): hanbinxy@163.com

E-mail: dyf@zju.edu.cn

Received May 12, 2014; revised January 20, 2015; published online March 15, 2015

\begin{abstract}
We are concerned with 3D incompressible generalized anisotropic NavierStokes equations with hyperdissipative term in horizontal variables. We prove that there exists a unique global solution for it with large initial data in anisotropic Besov space.
\end{abstract}

Keywords: anisotropic, hyper-dissipation, large data.

AMS Subject Classification: 35Q30; 35Q35; 76D03.

\section{Introduction}

In this paper, we are going to study the generalized 3-D incompressible anisotropic Navier-Stokes equations

$$
\left\{\begin{array}{l}
\partial_{t} u+u \cdot \nabla u+\nu_{h} D_{h}^{2 \alpha} u+\nu_{3} D_{3}^{2 \beta} u+\nabla \Pi=0 \\
\operatorname{div} u=0 \\
\left.u\right|_{t=0}=u_{0}
\end{array}\right.
$$

where $u$ and $\Pi$ represent the velocity and pressure of the fluids respectively. The viscosity coefficients $\nu_{h}, \nu_{3}>0 . D_{h}^{2 \alpha}$ and $D_{3}^{2 \beta}$ are two Fourier multipliers whose symbol is $h\left(\xi_{h}\right)=\left|\xi_{h}\right|^{2 \alpha}$ and $m(\xi)=\left|\xi_{3}\right|^{2 \beta}$ respectively, where $\xi_{h} \in \mathbb{R}^{2}$ and $\xi_{3} \in \mathbb{R}$. We consider this model in order to understand how much the dissipation we need to dominate the effects of convective term, so that the global solution of such an equation with large data exists. In this way we can understand 3-D Navier-Stokes equations better. In fact, there are some related works on this topic. We remark that when $\nu_{h}=\nu_{3}>0$ and $\alpha=\beta=1$, system (1.1) is nothing but the equations of classical Navier-Stokes equations. The global existence of strong solution for it is of course a great open problem, 
due to the super-critical nature of the equations. An anisotropic case $\alpha=\beta=1$ and $\nu_{h}>0, \nu_{3}=0$, has been studied first by J.Y. Chemin, B. Desjardins, I. Gallagher and E. Grenier in [3] and D. Iftimie in [6]. In [6], the author proved that such a system is locally wellposed for initial data in the anisotropic Sobolev space $\dot{H}^{0, s}$ for $s>\frac{1}{2}$. Moreover, it has also been proved that if the initial data are small enough in the sense that

$$
\left\|u_{0}\right\|_{L^{2}}^{\varepsilon}\left\|u_{0}\right\|_{\dot{H}^{0, s}}^{1-\varepsilon} \leq c \nu_{h}
$$

for some sufficiently small constant $c$ and $\varepsilon=s-\frac{1}{2}$, then the system (1.1) is global wellposed. Furthermore, T. Zhang in [11] proved that when $\alpha=\beta=1$ and $\nu_{h}>0, \nu_{3}=0$, system (1.1) is global wellposed provide the initial data $u_{0}$ satisfies

$$
C \nu_{h}\left\|u_{0}^{h}\right\|_{B^{0, \frac{1}{2}}} \exp \left\{C \nu_{h}^{-4}\left\|u_{0}^{3}\right\|_{B^{0, \frac{1}{2}}}^{4}+1\right\} \leq 1,
$$

where $B^{0, \frac{1}{2}}$ is the anisotropic Besov space with the regularity in vertical variable. Considering the periodic anisotropic Navier-Stokes equations, M. Paicu obtained global wellposedness in [9].

We note that, all above global results were obtained under the assumptions of small data in some sense. To get result for large data, people tried to strengthen the dissipative symbol $h\left(\xi_{h}\right)$ and $m\left(\xi_{3}\right)$, authors in [7] obtain the global regularity in critical and subcritical hyperdissipation regimes $h(\xi)=|\xi|^{\alpha}$ for $\alpha \geq \frac{N+2}{4}, N$ is the dimension of the space. This corresponds to the case $\alpha=\beta \geq \frac{N+2}{4}$ and $\nu_{h}=\nu_{3}>0$ in system (1.1). Tao [10] improved this slightly by establishing global regularity under a slightly weaker condition. He assumes that $h(\xi) \geq|\xi|^{\frac{N+2}{4}} / g(\xi)$ for all sufficiently large $\xi$ and some nondecreasing function $g: \mathbb{R}^{+} \rightarrow \mathbb{R}^{+}$such that $\int_{0}^{\infty} \frac{1}{s g(s)^{4}} d s=\infty$. While for the inhomogeneous flows, D. Fang and R. Zi [5] proved the global existence result for the hyperdissipative Navier-Stokes with initial data in subcritical Sobolev spaces.

Just as the classical Navier-Stokes system, (1.1) has a scaling. Indeed, under the following transformation

$$
\begin{aligned}
& u_{\lambda}^{h}(t, x)=\lambda^{2-\frac{1}{\alpha}} u^{h}\left(\lambda^{2} t, \lambda^{\frac{1}{\alpha}} x_{h}, \lambda^{\frac{1}{\beta}} x_{3}\right), \\
& u_{\lambda}^{3}(t, x)=\lambda^{2-\frac{1}{\beta}} u^{3}\left(\lambda^{2} t, \lambda^{\frac{1}{\alpha}} x_{h}, \lambda^{\frac{1}{\beta}} x_{3}\right),
\end{aligned}
$$

the scaling of dissipation term is the same as that of convection term. In this sense, the $L^{2}$ norm of $u_{\lambda}^{h}$ and $u_{\lambda}^{3}$ can be given by

$$
\begin{aligned}
& \left\|u_{\lambda}^{h}(t, x)\right\|_{L^{2}\left(\mathbb{R}^{3}\right)}=\lambda^{2-\frac{2}{\alpha}-\frac{1}{2 \beta}}\left\|u^{h}(t, x)\right\|_{L^{2}\left(\mathbb{R}^{3}\right)}, \\
& \left\|u_{\lambda}^{3}(t, x)\right\|_{L^{2}\left(\mathbb{R}^{3}\right)}=\lambda^{2-\frac{1}{\alpha}-\frac{3}{2 \beta}}\left\|u^{3}(t, x)\right\|_{L^{2}\left(\mathbb{R}^{3}\right)} .
\end{aligned}
$$

If one expect that the $L^{2}$ energy of $u^{h}$ be the critical nature of the equation, we need $2-\frac{2}{\alpha}-\frac{1}{2 \beta}=0$. Similarly, for $u^{3}$, we need $2-\frac{1}{\alpha}-\frac{3}{2 \beta}=0$. Obviously, we have the scaling index $\alpha=\beta=\frac{5}{4}$ and it is the case which has been studied in $[5,7,10]$. Here we want to investigate the effects of dissipation of horizontal variables and expect that $\beta=1$. Thus we obtain that $(\alpha, \beta)=\left(\frac{4}{3}, 1\right)$ is 
critical for the horizontal components $u^{h}$, and $(\alpha, \beta)=(2,1)$ is critical for the vertical component $u^{3}$. In this paper, taking advantage of the incompressible condition $\operatorname{div}_{\mathrm{h}} u^{h}=-\partial_{3} u^{3}$, we can get the global existence result when $(\alpha, \beta)=$ $\left(\frac{3}{2}, 1\right)$. One notes that $(\alpha, \beta)=\left(\frac{3}{2}, 1\right)$ is the supercritical index for $u^{3}$ but the subcritical index for $u^{h}$.

More generally, we prove that, for all $\alpha \geq \frac{3}{2}$ and $\beta=1$, system (1.1) admits a unique global solution for large initial data. It is obviously that for $\alpha \geq \frac{3}{2}$, $\beta=1,(1.1)$ satisfies the following basic energy estimate

$$
\|u(t)\|_{L^{2}}^{2}+2 \nu_{h} \int_{0}^{t}\left\|D_{h}^{\alpha} u\right\|_{L^{2}}^{2} d \tau+2 \nu_{3} \int_{0}^{t}\left\|\partial_{3} u\right\|_{L^{2}}^{2} d \tau=\left\|u_{0}\right\|_{L^{2}}^{2} .
$$

Our main result in this paper concerns the unique solvability of (1.1) with initial data in the anisotropic Besov spaces but without a smallness assumption on $u_{0}$.

Theorem 1. Let $s \geq 0, \alpha \geq \frac{3}{2}, \beta=1, u_{0} \in B^{0, s}\left(\mathbb{R}^{3}\right)$ with $\operatorname{div} u_{0}=0$. Then there exists a positive time $T$, such that (1.1) admits a local solution which satisfies

$$
u \in C\left([0, T] ; B^{0, s}\right) \quad \text { and } \quad \nabla_{h}^{\alpha} u \in \widetilde{L}^{2}\left(0, T ; B^{0, s}\right), \quad \partial_{3} u \in \widetilde{L}^{2}\left(0, T ; B^{0, s}\right) .
$$

More over, if $s \geq \frac{1}{2}$, then the local solution is unique and can be extended to the global one.

In Theorem 1 , we assume that $u_{0} \in B^{0, s}\left(\mathbb{R}^{3}\right)$ for all $s \geq 0$. Following the same method, we may find that such result also holds for anisotropic Sobolev space $H^{0, s}, s>0$. We assert that $s>0$ is necessary when $u_{0} \in H^{0, s}$, since Lemma 1 no longer valid for $s=0$.

Corollary 1. Let $s>0, \alpha \geq \frac{3}{2}, \beta=1, u_{0} \in H^{0, s}\left(\mathbb{R}^{3}\right)$ with $\operatorname{div} u_{0}=0$. Then there exists a positive time $T$, such that (1.1) admits a local solution which satisfies

$$
u \in C\left([0, T] ; H^{0, s}\right) \quad \text { and } \quad \nabla_{h}^{\alpha} u \in \widetilde{L}^{2}\left(0, T ; H^{0, s}\right), \quad \partial_{3} u \in \widetilde{L}^{2}\left(0, T ; H^{0, s}\right) .
$$

More over, if $s>\frac{1}{2}$, then the local solution is unique and can be extended to the global one.

The above results can be reached through an energy estimate. The advantage of hyper-disspation regime $h\left(\xi_{h}\right)=\left|\xi_{h}\right|^{\alpha}$ for $\alpha \geq \frac{3}{2}$ in our paper will be revealed in the estimate of convection term. More explicitly, we should split $\|u \cdot \nabla u\|_{L^{2}}$ into

$$
\|u \cdot \nabla u\|_{L^{2}} \leq\left\|u^{h} \cdot \nabla_{h} u\right\|_{L^{2}}+\left\|u^{3} \partial_{3} u\right\|_{L^{2}} .
$$

Since $\alpha \geq \frac{3}{2}$, the first term in the right hand side of above inequality can be estimated by

$$
\left\|u^{h} \cdot \nabla_{h} u\right\|_{L^{2}} \leq C\left\|\nabla_{h}^{\alpha} u\right\|_{L^{2}}^{\frac{3}{2 \alpha}}\left\|\partial_{3} u\right\|_{L^{2}}^{\frac{1}{2}-\frac{1}{2 \alpha}}\left\|\partial_{3} \nabla_{h}^{\alpha} u\right\|_{L^{2}}^{\frac{1}{2 \alpha}}
$$


One can see the details in (4.3). Hence one can use Young's inequality to close the energy estimate(see the details in Lemma 2). We observe that when $\alpha=1$, the convection term was bounded by

$$
\|u \cdot \nabla u\|_{L^{2}} \leq C\|u\|_{L^{2}}^{\frac{1}{4}}\|\nabla u\|_{L^{2}}\left\|\nabla^{2} u\right\|_{L^{2}}^{\frac{3}{4}}
$$

in three dimensional space, to close the energy estimate, the smallness condition on $u_{0}$ is necessary. The second term is more delicate, we will use divergence free on $u$ to write

$$
\partial_{3} u^{3}=-\operatorname{div}_{h} u^{h}
$$

Another important estimate in our paper is the $B^{0, s}$ energy estimate of convection term when proving the blow-up criterion. It reads that

$$
\begin{aligned}
\left|\left(\Delta_{k}^{v}(u \cdot \nabla u) \mid \Delta_{k}^{v} u\right)_{L^{2}}\right| & \leq C d_{k} 2^{-2 k s}\|u\|_{L_{v}^{\infty}\left(L_{h}^{2}\right)}^{\frac{1}{2}}\left\|\nabla_{h} u\right\|_{L_{v}^{\infty}\left(L_{h}^{2}\right)}^{\frac{1}{2}}\|u\|_{B^{0, s}}^{\frac{1}{2}}\left\|\nabla_{h} u\right\|_{B^{0, s}}^{\frac{3}{2}} \\
& +C d_{k} 2^{-2 k s}\|u\|_{L_{v}^{\infty}\left(L_{h}^{2}\right)}^{\frac{1}{2}}\left\|\nabla_{h} u\right\|_{L_{v}^{\infty}\left(L_{h}^{2}\right)}^{\frac{1}{2}}\|u\|_{B^{0, s}}^{\frac{3}{2}}\left\|\nabla_{h} u\right\|_{B^{0, s}}^{\frac{1}{2}} \\
& +C d_{k} 2^{-2 k s}\left\|\nabla_{h} u\right\|_{L_{v}^{\infty}\left(L_{h}^{2}\right)}\|u\|_{B^{0, s}}\left\|\nabla_{h} u\right\|_{B^{0, s}}
\end{aligned}
$$

for all $s \geq 0$. The similar estimate in Sobolev space $H^{s}$ was proved in [8] (Lemma 3.1). The main difficulty in this estimate is how to bound the $u^{3} \partial_{3} u$ by the horizonal derivative $\nabla_{h} u$. To overcome it, we have to use Bony decomposition in vertical direction. We may find that $u^{3} \partial_{3} u$ can be decomposed by

$$
\Delta_{k}^{v}\left(\sum_{k^{\prime} \geq k-N_{0}} S_{k^{\prime}+2}^{v}\left(\partial_{3} u\right) \Delta_{k^{\prime}}^{v} u^{3}\right)+\Delta_{k}^{v}\left(\sum_{\left|k^{\prime}-k\right| \leq N_{0}} S_{k^{\prime}-2}^{v} u^{3} \partial_{3} \Delta_{k^{\prime}}^{v} u\right)
$$

The first term of (1.3) can be dealt by Bernstein inequality since the spectrum support of $\Delta_{k^{\prime}}^{v} u^{3}$ is contained in an annular. Formally, when such term estimated in Lebesgue space, we can transfer the operator $\partial_{3}$ from $S_{k^{\prime}+2}^{v}\left(\partial_{3} u\right)$ to $\Delta_{k^{\prime}}^{v} u^{3}$. Then the divergence free on $u$ enable us to get the desired estimate. But it can not be used in the second term. We will use a commutator estimate and integration by parts to complete the estimate on the second term. One can see the details in Lemma 1.

The rest of the paper is structured as follows. In Section 2, we present the definition of anisotropic Littlewood-Paley decomposition and the theory of anisotropic Besov space. In Section 3, we show the local existence result and prove a blow-up criterion of (1.1). Finally, we present a high order energy estimate of (1.1) and complete the proof the existence of the global solution.

\section{Anisotropic Littlewood-Paley Theory}

Because the space we will use is anisotropic Besov spaces, so, in this section, we recall the Hölder and Young's inequalities in the frame of anisotropic Lebesgue spaces at first. 
Proposition 1. 1) Let $f \in L_{v}^{r^{\prime}}\left(L_{h}^{p^{\prime}}\right), g \in L_{v}^{r^{\prime \prime}}\left(L_{h}^{p^{\prime \prime}}\right)$ for $1 \leq r, r^{\prime}, r^{\prime \prime}, p, p^{\prime}, p^{\prime \prime} \leq$ $\infty$, then $f g \in L_{v}^{r}\left(L_{h}^{p}\right)$. And the following inequality holds

$$
\|f g\|_{L_{v}^{r}\left(L_{h}^{p}\right)} \leq C\|f\|_{L_{v}^{r^{\prime}}\left(L_{h}^{p^{\prime}}\right)}\|g\|_{L_{v}^{r^{\prime \prime}}\left(L_{h}^{p^{\prime \prime}}\right)},
$$

where $\frac{1}{r}=\frac{1}{r^{\prime}}+\frac{1}{r^{\prime \prime}}$ and $\frac{1}{p}=\frac{1}{p^{\prime}}+\frac{1}{p^{\prime \prime}}$.

2) Let $f \in L_{v}^{r^{\prime}}\left(L_{h}^{p^{\prime}}\right), g \in L_{v}^{r^{\prime \prime}}\left(L_{h}^{p^{\prime \prime}}\right)$ for $1 \leq r, r^{\prime}, r^{\prime \prime}, p, p^{\prime}, p^{\prime \prime} \leq \infty$, then $f \star g \in L_{v}^{r}\left(L_{h}^{p}\right)$. And the following inequality holds

$$
\|f \star g\|_{L_{v}^{r}\left(L_{h}^{p}\right)} \leq C\|f\|_{L_{v}^{r^{\prime}}\left(L_{h}^{p^{\prime}}\right)}\|g\|_{L_{v}^{r^{\prime \prime}}\left(L_{h}^{p^{\prime \prime}}\right)},
$$

where $1+\frac{1}{r}=\frac{1}{r^{\prime}}+\frac{1}{r^{\prime \prime}}$ and $1+\frac{1}{p}=\frac{1}{p^{\prime}}+\frac{1}{p^{\prime \prime}}$.

To recall the definition of the anisotropic spaces, we have to introduce an anisotropic dyadic decomposition of Fourier variables, which is called the Anisotropic Littlewood-Paley decomposition. Let us briefly explain how it may be built.

Let $(\chi(r), \varphi(r))$ be a couple of $C^{\infty}$ functions satisfying

$$
\operatorname{Supp} \chi \subset\left\{|r| \leq \frac{4}{3}\right\}, \quad \operatorname{Supp} \varphi \subset\left\{\frac{3}{4} \leq|r| \leq \frac{8}{3}\right\}, \quad \chi(r)+\sum_{q \in \mathbb{N}} \varphi\left(2^{-q} r\right)=1
$$

for any $r \in \mathbb{R}$. Let $\varphi_{q}(r)=\varphi\left(2^{-q} r\right), h_{q}=\mathcal{F}^{-1} \varphi_{q}$, and $\tilde{h}=\mathcal{F}^{-1} \chi$. The dyadic blocks in vertical frequencies are defined by

$$
\begin{gathered}
\Delta_{q}^{v} u=0 \quad \text { if } q<-1, \quad \Delta_{-1}^{v} u=\chi\left(D_{3}\right) u=\int_{\mathbb{R}} \tilde{h}\left(y_{3}\right) u\left(x_{h}, x_{3}-y_{3}\right) d y_{3}, \\
\Delta_{q}^{v} u=\varphi\left(2^{-q} D_{3}\right) u=\int_{\mathbb{R}} h_{q}\left(y_{3}\right) u\left(x_{h}, x_{3}-y_{3}\right) d y_{3} \quad \text { if } q \geq 0 .
\end{gathered}
$$

It is also convenient to introduce the following low frequency cut-off:

$$
S_{q}^{v} u=\sum_{p \leq q-1} \Delta_{p}^{v} u
$$

Of course, $S_{0}^{v} u=\Delta_{-1}^{v} u$. The anisotropic Littlewood-Paley decomposition has a nice property of quasi-orthogonality:

$$
\left.\Delta_{p}^{v} \Delta_{q}^{v} u \equiv 0 \quad \text { if }|p-q| \geq 2 \quad \text { and } \quad \Delta_{p}^{v}\left(S_{q-1}^{v} u \Delta_{q}^{v} u\right)\right) \equiv 0 \quad \text { if }|p-q| \geq 5 .
$$

Let us give the definition of nonhomogeneous anisotropic Besov spaces.

DeFinition 1. Let $u$ be a tempered distribution, we set

$$
\|u\|_{B_{2, r}^{0, s}}=\left(\sum_{q \geq-1} 2^{r q s}\left\|\Delta_{q}^{v} u\right\|_{L^{2}}^{r}\right)^{\frac{1}{r}}
$$

with $s \in \mathbb{R}$. We then define the anisotropic Besov space

$$
B_{2, r}^{0, s}=\left\{u \in \mathcal{S}^{\prime}\left(\mathbb{R}^{3}\right),\|u\|_{B_{2, r}^{0, s}}<\infty\right\} .
$$


Hereafter, we denote that $B_{2,2}^{0, s}:=H^{0, s}, B_{2,1}^{0, s}:=B^{0, s} . H^{0, s}$ is the anisotropic Sobolev space. The following two key estimates based on the divergence-free condition.

Proposition 2. There exists a positive constant $C$, such that for every divergence-free vector field $u=\left(u^{1}, u^{2}, u^{3}\right)$,

$$
\left\|\nabla u^{3}\right\|_{B^{0, s}} \leq C\left\|\nabla_{h} u\right\|_{B^{0, s}}, \quad\left\|\Delta_{k}^{v} u\right\|_{L_{v}^{2}\left(L_{h}^{4}\right)} \leq d_{k} 2^{-k s}\|u\|_{B^{0, s}}^{\frac{1}{2}}\left\|\nabla_{h} u\right\|_{B^{0, s}}^{\frac{1}{2}},
$$

where $d_{k}$ stands for a generic positive sequence such that $\sum_{k \geq-1} d_{k} \leq 1$.

Proof. The divergence-free condition implies that

$$
\nabla u^{3}=\left(\nabla_{h} u^{3},-\operatorname{div}_{h} u^{h}\right),
$$

from which the first estimate directly follows. To prove the second estimate, we write

$$
\begin{aligned}
\left\|\Delta_{k}^{v} u\right\|_{L_{v}^{2} L_{h}^{4}} & \leq C\left\|\Delta_{k}^{v}\right\|_{L^{2}\left(\mathbb{R}^{3}\right)}^{\frac{1}{2}}\left\|\Delta_{k}^{v} \nabla_{h} u\right\|_{L^{2}\left(\mathbb{R}^{3}\right)}^{\frac{1}{2}} \\
& \leq C\left(2^{-\frac{k s}{2}} d_{k}^{\frac{1}{2}}\|u\|_{B^{0, s}}^{\frac{1}{2}}\right)\left(2^{-\frac{k s}{2}} d_{k}^{\frac{1}{2}}\left\|\nabla_{h} u\right\|_{B^{0, s}}^{\frac{1}{2}}\right) \\
& \leq C 2^{-k s} d_{k}\|u\|_{B^{0, s}}^{\frac{1}{2}}\left\|\nabla_{h} u\right\|_{B^{0, s}}^{\frac{1}{2}} .
\end{aligned}
$$

The following lemma is the core of the proof of Theorem 1 .

Lemma 1. Let $\alpha \geq \frac{3}{2}$, then for any real number $s \geq 0$, there exists a positive constant $C_{s}$ such that, for any $u$, $v$, with $u$ divergence free, we have

$$
\begin{aligned}
& \left|\left(\Delta_{k}^{v}(u \cdot \nabla v) \mid \Delta_{k}^{v} v\right)_{L^{2}}\right| \leq C_{s} d_{k}^{2} 2^{-2 k s}\|u\|_{L_{v}^{\infty}\left(L_{h}^{2}\right)}\left(\|v\|_{B^{0, s}}^{1-\frac{5}{4 \alpha}}\left\|\nabla_{h}^{\alpha} v\right\|_{B^{0, s}}^{\frac{5}{4 \alpha}}+\|v\|_{B^{0, s}}\right) \\
& \quad \times\left(\|v\|_{B^{0, s}}^{1-\frac{1}{\alpha}}\left\|\nabla_{h}^{\alpha} v\right\|_{B^{0, s}}^{\frac{1}{\alpha}}+\|v\|_{B^{0, s}}\right)+C_{s} d_{k}^{2} 2^{-2 k s}\|v\|_{L_{v}^{\infty}\left(L_{h}^{2}\right)} \\
& \quad \times\left(\|u\|_{B^{0, s}}^{1-\frac{5}{4 \alpha}}\left\|\nabla_{h}^{\alpha} u\right\|_{B^{0, s}}^{\frac{5}{4 \alpha}}+\|u\|_{B^{0, s}}\right)\left(\|v\|_{B^{0, s}}^{1-\frac{1}{\alpha}}\left\|\nabla_{h}^{\alpha} v\right\|_{B^{0, s}}^{\frac{1}{\alpha}}+\|v\|_{B^{0, s}}\right),
\end{aligned}
$$

where $(\cdot \mid \cdot)_{L^{2}}$ denotes the $L^{2}$ inner product of two functions. In particular, if $v=u$, there holds

$$
\begin{aligned}
& \left|\left(\Delta_{k}^{v}(u \cdot \nabla u) \mid \Delta_{k}^{v} u\right)_{L^{2}}\right| \leq C_{s} d_{k}^{2} 2^{-2 k s}\left(\|u\|_{L_{v}^{\infty}\left(L_{h}^{2}\right)}^{\frac{1}{2}}\left\|\nabla_{h} u\right\|_{L_{v}^{\infty}\left(L_{h}^{2}\right)}^{\frac{1}{2}}\|u\|_{B^{0, s}}^{\frac{1}{2}}\left\|\nabla_{h} u\right\|_{B^{0, s}}^{\frac{3}{2}}\right. \\
& \quad+\|u\|_{L_{v}^{\infty}\left(L_{h}^{2}\right)}^{\frac{1}{2}}\left\|\nabla_{h} u\right\|_{L_{v}^{\infty}\left(L_{h}^{2}\right)}^{\frac{1}{2}}\|u\|_{B^{0, s}}^{\frac{3}{2}}\left\|\nabla_{h} u\right\|_{B^{0, s}}^{\frac{1}{2}} \\
& \left.\quad+\left\|\nabla_{h} u\right\|_{L_{v}^{\infty}\left(L_{h}^{2}\right)}\|u\|_{B^{0, s}}\left\|\nabla_{h} u\right\|_{B^{0, s}}\right) .
\end{aligned}
$$

Proof. Let us define

$$
F_{k}^{h}:=\Delta_{k}^{v}\left(u^{h} \cdot \nabla_{h} v\right) \quad \text { and } \quad F_{k}^{v}:=\Delta_{k}^{v}\left(u^{3} \partial_{3} v\right) .
$$

The term $\left(F_{k}^{h} \mid \Delta_{k}^{v} v\right)_{L^{2}}$ and $\left(F_{k}^{v} \mid \Delta_{k}^{v} v\right)_{L^{2}}$ are estimated in different ways. Let us start by proving the result for $F_{k}^{h}$. Applying Bony decomposition in vertical variable, we have

$$
\begin{aligned}
F_{k}^{h} & =\Delta_{k}^{v}\left(T_{u^{h}}^{v} \nabla_{h} v+T_{\nabla_{h} v}^{v} u^{h}+R^{v}\left(u^{h}, \nabla_{h} v\right)\right. \\
& :=F_{k}^{h, 1}+F_{k}^{h, 2}+F_{k}^{h, 3},
\end{aligned}
$$


where

$$
\begin{aligned}
& F_{k}^{h, 1}=\Delta_{k}^{v} \sum_{\left|k-k^{\prime}\right| \leq N_{0}}\left(S_{k^{\prime}-1}^{v} u^{h} \Delta_{k^{\prime}}^{v} \nabla_{h} v\right), \\
& F_{k}^{h, 2}=\Delta_{k}^{v} \sum_{\left|k-k^{\prime}\right| \leq N_{0}}\left(S_{k^{\prime}-1}^{v} \nabla_{h} v \Delta_{k^{\prime}}^{v} u^{h}\right), \quad F_{k}^{h, 3}=\Delta_{k}^{v} \sum_{k^{\prime}>k-N_{0}}\left(\Delta_{k^{\prime}}^{v} \nabla_{h} v \widetilde{\Delta}_{k^{\prime}}^{v} u^{h}\right)
\end{aligned}
$$

According to Proposition 1, together with interpolation inequality, we get

$$
\begin{aligned}
\left(F_{k}^{h, 1} \mid \Delta_{k}^{v} v\right)_{L^{2}} & =C \sum_{\left|k-k^{\prime}\right| \leq N_{0}}\left\|S_{k^{\prime}-1}^{v} u^{h}\right\|_{L_{v}^{\infty}\left(L_{h}^{2}\right)}\left\|\Delta_{k^{\prime}}^{v} \nabla_{h} v\right\|_{L_{v}^{2}\left(L_{h}^{\frac{8}{3}}\right)}\left\|\Delta_{k}^{v} v\right\|_{L_{v}^{2}\left(L_{h}^{8}\right)} \\
\leq & C\|u\|_{L_{v}^{\infty}\left(L_{h}^{2}\right)} \sum_{\left|k^{\prime}-k\right| \leq N_{0}}\left\|\Delta_{k^{\prime}}^{v} v\right\|_{L^{2}}^{1-\frac{5}{4 \alpha}}\left\|\Delta_{k^{\prime}}^{v} \nabla_{h}^{\alpha} v\right\|_{L^{2}}^{\frac{5}{4 \alpha}}\left\|\Delta_{k}^{v} v\right\|_{L_{v}^{2}\left(H^{1}\left(\mathbb{R}^{2}\right)\right)} \\
\leq & C\|u\|_{L_{v}^{\infty}\left(L_{h}^{2}\right)} \sum_{\left|k^{\prime}-k\right| \leq N_{0}}\left\|\Delta_{k^{\prime}}^{v} v\right\|_{L^{2}}^{1-\frac{5}{4 \alpha}}\left\|\Delta_{k^{\prime}}^{v} \nabla_{h}^{\alpha} v\right\|_{L^{2}}^{\frac{5}{4 \alpha}} \\
& \times\left(\left\|\Delta_{k}^{v} v\right\|_{L^{2}}^{1-\frac{1}{\alpha}}\left\|\Delta_{k}^{v} \nabla_{h}^{\alpha} v\right\|_{L^{2}}^{\frac{1}{\alpha}}+\left\|\Delta_{k}^{v} v\right\|_{L^{2}}\right) \\
\leq & C d_{k}^{2} 2^{-2 k s}\|u\|_{L_{v}^{\infty}\left(L_{h}^{2}\right)}\|v\|_{B^{0, s}}^{1-\frac{5}{4 \alpha}}\left\|\nabla_{h}^{\alpha} v\right\|_{B^{0, s}}^{\frac{5}{4 \alpha}}\left(\|v\|_{B^{0, s}}^{1-\frac{1}{\alpha}}\left\|\nabla_{h}^{\alpha} v\right\|_{B^{0, s}}^{\frac{1}{\alpha}}+\|v\|_{B^{0, s}}\right) .
\end{aligned}
$$

Similarly computation implies

$$
\begin{aligned}
\left(F_{k}^{h, 2} \mid \Delta_{k}^{v} v\right)_{L^{2}} & \left\|S_{\left|k-k^{\prime}\right| \leq N_{0}}^{v} \nabla_{k^{\prime}-1} \nabla_{h} v\right\|_{L_{v}^{\infty}\left(H^{-1}\left(\mathbb{R}^{2}\right)\right)}\left\|\Delta_{k^{\prime}}^{v} u^{h}\right\|_{L_{v}^{2}\left(H^{\left.\frac{5}{4}\left(\mathbb{R}^{2}\right)\right)}\right.}\left\|\Delta_{k}^{v} v\right\|_{L_{v}^{2}\left(H^{1}\left(\mathbb{R}^{2}\right)\right)} \\
\leq & C\|v\|_{L_{v}^{\infty}\left(L_{h}^{2}\right)} \sum_{\left|k^{\prime}-k\right| \leq N_{0}}\left(\left\|\Delta_{k^{\prime}}^{v} u\right\|_{L^{2}}^{1-\frac{5}{4 \alpha}}\left\|\Delta_{k^{\prime}}^{v} \nabla_{h}^{\alpha} u\right\|_{L^{2}}^{\frac{5}{4 \alpha}}+\left\|\Delta_{k^{\prime}}^{v} u\right\|_{L^{2}}\right) \\
& \times\left(\left\|\Delta_{k}^{v} v\right\|_{L^{2}}^{1-\frac{1}{\alpha}}\left\|\Delta_{k}^{v} \nabla_{h}^{\alpha} v\right\|_{L^{2}}^{\frac{1}{\alpha}}+\left\|\Delta_{k}^{v} v\right\|_{L^{2}}\right) \\
\leq & C_{s} d_{k}^{2} 2^{-2 k s}\|v\|_{L_{v}^{\infty}\left(L_{h}^{2}\right)}\left(\|u\|_{B^{0, s}}^{1-\frac{5}{4 \alpha}}\left\|\nabla_{h}^{\alpha} u\right\|_{B^{0, s}}^{\frac{5}{4 \alpha}}+\|u\|_{B^{0, s}}\right) \\
& \times\left(\|v\|_{B^{0, s}}^{1-\frac{1}{\alpha}}\left\|\nabla_{h}^{\alpha} v\right\|_{B^{0, s}}^{\frac{1}{\alpha}}+\|v\|_{B^{0, s}}\right) .
\end{aligned}
$$

While for $F_{k}^{h, 3}$, by Proposition 1 and interpolation inequality, we have

$$
\begin{aligned}
& \left(F_{k}^{h, 3} \mid \Delta_{k}^{v} v\right)_{L^{2}}\left\|\Delta_{k^{\prime} \geq k-N_{0}}^{v} u_{k^{\prime}}^{h}\right\|_{L_{v}^{\infty}\left(L_{h}^{2}\right)}\left\|\widetilde{\Delta}_{k^{\prime}}^{v} \nabla_{h} v\right\|_{L_{v}^{2}\left(L_{h}^{\frac{8}{3}}\right)}\left\|\Delta_{k}^{v} v\right\|_{L_{v}^{2}\left(L_{h}^{8}\right)} \\
& \quad \leq C \sum_{L_{v}^{\infty}\left(L_{h}^{2}\right)} \sum_{k^{\prime} \geq k-N_{0}}\left\|\widetilde{\Delta}_{k^{\prime}}^{v} v\right\|_{L^{2}}^{1-\frac{5}{4 \alpha}}\left\|\widetilde{\Delta}_{k^{\prime}}^{v} \nabla_{h}^{\alpha} v\right\|_{L^{2}}^{\frac{5}{4 \alpha}}
\end{aligned}
$$




$$
\begin{aligned}
& \times\left(\left\|\Delta_{k}^{v} v\right\|_{L^{2}}^{1-\frac{1}{\alpha}}\left\|\Delta_{k}^{v} \nabla_{h}^{\alpha} v\right\|_{L^{2}}^{\frac{1}{\alpha}}+\left\|\Delta_{k}^{v} v\right\|_{L^{2}}\right) \\
\leq & C d_{k} 2^{-2 k s}\|u\|_{L_{v}^{\infty}\left(L_{h}^{2}\right)} \sum_{k^{\prime} \geq k-N_{0}} 2^{\left(k-k^{\prime}\right) s} 2^{k^{\prime} s}\left\|\widetilde{\Delta}_{k^{\prime}}^{v} v\right\|_{L^{2}}^{1-\frac{5}{4 \alpha}}\left\|\widetilde{\Delta}_{k^{\prime}}^{v} \nabla_{h}^{\alpha} v\right\|_{L^{2}}^{\frac{5}{4 \alpha}} \\
& \times\left(\|v\|_{B^{0, s}}^{1-\frac{1}{\alpha}}\left\|\nabla_{h}^{\alpha} v\right\|_{B^{0, s}}^{\frac{1}{\alpha}}+\|v\|_{B^{0, s}}\right) \\
\leq & C d_{k}^{2} 2^{-2 k s}\|u\|_{L_{v}^{\infty}\left(L_{h}^{2}\right)}\|v\|_{B^{0, s}}^{1-\frac{5}{4 \alpha}}\left\|\nabla_{h}^{\alpha} v\right\|_{B^{0, s}}^{\frac{5}{4 \alpha}}\left(\|v\|_{B^{0, s}}^{1-\frac{1}{\alpha}}\left\|\nabla_{h}^{\alpha} v\right\|_{B^{0, s}}^{\frac{1}{\alpha}}+\|v\|_{B^{0, s}}\right),
\end{aligned}
$$

where we have used $s \geq 0$ in the last inequality. So (2.7), (2.8) and (2.9) imply that (2.5) is proved for the term $\left(F_{k}^{h} \mid \Delta_{k}^{v} v\right)_{L^{2}}$. To estimate the term $\left(F_{k}^{v} \mid \Delta_{k}^{v} v\right)_{L^{2}}$, let us again use Bony decomposition in the vertical variable:

$$
\Delta_{k}^{v}\left(u^{3} \partial_{3} v\right)=F_{k}^{v, 1}+F_{k}^{v, 2}
$$

with

$$
F_{k}^{v, 1}=\Delta_{k}^{v} \sum_{k^{\prime} \geq k-N_{0}} S_{k^{\prime}+2}^{v}\left(\partial_{3} v\right) \Delta_{k^{\prime}}^{v} u^{3}, \quad F_{k}^{v, 2}=\Delta_{k}^{v} \sum_{\left|k^{\prime}-k\right| \leq N_{0}} S_{k^{\prime}-1}^{v} u^{3} \partial_{3} \Delta_{k^{\prime}}^{v} v .
$$

Clearly, we have

$$
\left|\left(F_{k}^{v, 1} \mid \Delta_{k}^{v} v\right)_{L^{2}}\right| \leq C\left\|\Delta_{k}^{v} v\right\|_{L_{v}^{2}\left(L_{h}^{8}\right)}\left\|F_{k}^{v, 1}\right\|_{L_{v}^{2}\left(L_{h}^{\frac{8}{7}}\right)} .
$$

Bernstein's inequality, along with Proposition 1 yields

$$
\begin{aligned}
\left\|F_{k}^{v, 1}\right\|_{L_{v}^{2}\left(L_{h}^{\frac{8}{7}}\right)} & \leq C \sum_{k^{\prime} \geq k-N_{0}} 2^{k^{\prime}}\left\|S_{k^{\prime}+2}^{v} v\right\|_{L_{v}^{\infty}\left(L_{h}^{2}\right)}\left\|\Delta_{k^{\prime}}^{v} u^{3}\right\|_{L_{v}^{2}\left(L_{h}^{\frac{8}{3}}\right)} \\
& \leq C\|v\|_{L_{v}^{\infty}\left(L_{h}^{2}\right)} \sum_{k^{\prime} \geq k-N_{0}} 2^{k^{\prime}}\left\|\Delta_{k^{\prime}}^{v} u^{3}\right\|_{L_{v}^{2}\left(L_{h}^{\frac{8}{3}}\right)} .
\end{aligned}
$$

Here we are going to use in a crucial way the fact that $u$ is a divergence free:

$$
\partial_{3} u^{3}=-\operatorname{div}_{h} u^{h} .
$$

Thus, we infer that

$$
\begin{aligned}
\left\|F_{k}^{v, 1}\right\|_{L_{v}^{2}\left(L_{h}^{\frac{8}{7}}\right)} \leq & C\|v\|_{L_{v}^{\infty}\left(L_{h}^{2}\right)} \sum_{\substack{k^{\prime} \geq k-N_{0} \\
k^{\prime} \geq 0}}\left\|\Delta_{k^{\prime}}^{v} \nabla_{h} u^{h}\right\|_{L_{v}^{2}\left(L_{h}^{\frac{8}{3}}\right)} \\
& +C\|v\|_{L_{v}^{\infty}\left(L_{h}^{2}\right)}\left\|\Delta_{-1}^{v} u^{3}\right\|_{L_{v}^{2}\left(L_{h}^{\frac{8}{3}}\right)} \\
\leq & C d_{k} 2^{-k s}\|v\|_{L_{v}^{\infty}\left(L_{h}^{2}\right)}\left(\|u\|_{B^{0, s}}^{1-\frac{5}{4 \alpha}}\left\|\nabla_{h}^{\alpha} u\right\|_{B^{0, s}}^{\frac{5}{4 \alpha}}+\|u\|_{B^{0, s}}\right) .
\end{aligned}
$$

Here we use the fact that when $k^{\prime}=-1$, the number of $k$ is finite. Then making use of (2.12) in (2.10), one can deduce that

$$
\begin{aligned}
\left|\left(F_{k}^{v, 1} \mid \Delta_{k}^{v} v\right)_{L^{2}}\right| \leq & C d_{k}^{2} 2^{-2 k s}\|v\|_{L_{v}^{\infty}\left(L_{h}^{2}\right)}\left(\|u\|_{B^{0, s}}^{1-\frac{5}{4 \alpha}}\left\|\nabla_{h}^{\alpha} u\right\|_{B^{0, s}}^{\frac{5}{4 \alpha}}+\|u\|_{B^{0, s}}\right) \\
& \times\left(\|v\|_{B^{0, s}}^{1-\frac{1}{\alpha}}\left\|\nabla_{h}^{\alpha} v\right\|_{B^{0, s}}^{\frac{1}{\alpha}}+\|v\|_{B^{0, s}}\right) .
\end{aligned}
$$


Up to that point, we did not actually use the energy estimate, but only laws of product or Sobolev embedding. The estimate of the term $\left(F_{k}^{v, 2} \mid \Delta_{k}^{v} v\right)_{L^{2}\left(\mathbb{R}^{3}\right)}$ will use in a crucial way the structure of the nonlinearity. First of all, following a computation done in [4], we get that

$$
\left(F_{k}^{v, 2} \mid \Delta_{k}^{v} v\right)_{L^{2}}=\left(S_{k}^{v} u^{3} \partial_{3} \Delta_{k}^{v} v \mid \Delta_{k}^{v} v\right)_{L^{2}}+R_{k}(u, v)
$$

with $R_{k}(u, v)$ defined by

$$
\begin{aligned}
& \sum_{\left|k-k^{\prime}\right| \leq N_{0}}\left(\left[\Delta_{k}^{v}, S_{k^{\prime}-2} u^{3}\right] \partial_{3} \Delta_{k^{\prime}}^{v} v \mid \Delta_{k}^{v} v\right)_{L^{2}} \\
& \left.+\sum_{\left|k-k^{\prime}\right| \leq N_{0}}\left(S_{k}^{v}-S_{k^{\prime}-2}^{v}\right) u^{3} \partial_{3} \Delta_{k^{\prime}}^{v} v \mid \Delta_{k}^{v} v\right)_{L^{2}}
\end{aligned}
$$

Then, using an integration by parts, we infer that

$$
\left(S_{k}^{v} u^{3} \partial_{3} \Delta_{k}^{v} v \mid \Delta_{k}^{v} v\right)_{L^{2}}=-\frac{1}{2}\left(S_{k}^{v}\left(\partial_{3} u^{3}\right) \Delta_{k}^{v} v \mid \Delta_{k}^{v} v\right)_{L^{2}}
$$

Thanks to the fact that $u$ is divergence free, $\left(S_{k}^{v} u^{3} \partial_{3} \Delta_{k}^{v} v \mid \Delta_{k}^{v} v\right)_{L^{2}}$ can be estimated as the term $\left(F_{k}^{h} \mid \Delta_{k}^{v}\right)_{L^{2}}$ which appeared above. The third term $\left(S_{k}^{v}-\right.$ $\left.\left.S_{k^{\prime}-1}^{v}\right) u^{3} \partial_{3} \Delta_{k^{\prime}}^{v} v \mid \Delta_{k}^{v} v\right)_{L^{2}}$ can be estimated exactly as the term $F_{k}^{v, 1}$. So we will mainly give the details of the proof of $\left(\left[\Delta_{k}^{v}, S_{k^{\prime}-1}^{v} u^{3}\right] \partial_{3} \Delta_{k^{\prime}}^{v} v \mid \Delta_{k}^{v} v\right)_{L^{2}}$. Note that for any function $w$,

$$
\begin{aligned}
{\left[\Delta_{k}^{v}, S_{k^{\prime}-1} u^{3}\right] w(x)=2^{k} } & \int_{\mathbb{R}} h\left(2^{k} y_{3}\right)\left(S_{k^{\prime}-1}^{v} u^{3}\left(x_{h}, x_{3}\right)\right. \\
& \left.-S_{k^{\prime}-1}^{v} u^{3}\left(x_{h}, x_{3}-y_{3}\right)\right) w\left(x_{h}, x_{3}-y_{3}\right) d y_{3} .
\end{aligned}
$$

Writing a Taylor formula, we get that

$$
\begin{aligned}
& {\left[\Delta_{k}^{v}, S_{k^{\prime}-1} u^{3}\right] w(x)} \\
& \quad=\int_{\mathbb{R} \times[0,1]} \tilde{h}\left(2^{k} y_{3}\right)\left(S_{k^{\prime}-1}^{v} \partial_{3} u^{3}\right)\left(x_{h}, x_{3}+t\left(x_{3}-y_{3}\right)\right) w\left(x_{h}, x_{3}-y_{3}\right) d y_{3} d t
\end{aligned}
$$

with $\widetilde{h}(z)=z h(z)$. Thanks to the fact that $u$ is a divergence free, we get

$$
\begin{aligned}
& {\left[\Delta_{k}^{v}, S_{k^{\prime}-1}^{v} u^{3}\right] w(x)} \\
& \quad=-\int_{\mathbb{R} \times[0,1]} \tilde{h}\left(2^{k} y_{3}\right)\left(S_{k^{\prime}-1}^{v} \operatorname{div}_{h} u^{h}\right)\left(x_{h}, x_{3}+t\left(x_{3}-y_{3}\right)\right) w\left(x_{h}, x_{3}-y_{3}\right) d y_{3} d t .
\end{aligned}
$$

Now, applying the rules of product of Sobolev spaces on $\mathbb{R}^{2}$, one can obtain that

$$
\begin{aligned}
& \left\|\left[\Delta_{k}^{v}, S_{k^{\prime}-1}^{v} u^{3}\right] w(x)\right\|_{H^{-1}\left(\mathbb{R}^{2}\right)} \\
& \quad \leq C \int_{\mathbb{R}}\left|\widetilde{h}\left(2^{k} y_{3}\right)\right|\left\|S_{k^{\prime}-1}^{v} \operatorname{div}_{h} u^{h}\right\|_{L_{v}^{\infty}\left(H^{-1}\left(\mathbb{R}^{2}\right)\right)}\left\|w\left(\cdot, x_{3}-y_{3}\right)\right\|_{H^{\frac{5}{4}\left(\mathbb{R}^{2}\right)}} d y_{3} .
\end{aligned}
$$


Applying Hölder inequality in (2.15), we deduce that

$$
\left\|\left[\Delta_{k}^{v}, S_{k^{\prime}-1}^{v} u^{3}\right] w(x)\right\|_{L_{v}^{2}\left(H^{-1}\left(\mathbb{R}^{2}\right)\right)} \leq C 2^{-k}\left\|S_{k^{\prime}-1}^{v} u^{h}\right\|_{L_{v}^{\infty}\left(L_{h}^{2}\right)}\|w\|_{L_{v}^{2}\left(H^{\left.\frac{5}{4}\left(\mathbb{R}^{2}\right)\right)}\right.} .
$$

Setting $w=\partial_{3} \Delta_{k^{\prime}}^{v} v$ in (2.16), by interpolation inequality, we finally get that

$$
\begin{aligned}
& \sum_{\left|k-k^{\prime}\right| \leq N_{0}}\left(\left[\Delta_{k}^{v}, S_{k^{\prime}-1}^{v} u^{3}\right] \partial_{3} \Delta_{k^{\prime}}^{v} v \mid \Delta_{k}^{v} v\right)_{L^{2}} \leq C d_{k}^{2} 2^{-2 k s}\|u\|_{L_{v}^{\infty}\left(L_{h}^{2}\right)} \\
& \quad \times\left(\|v\|_{B^{0, s}}^{1-\frac{5}{4 \alpha}}\left\|\nabla_{h}^{\alpha} v\right\|_{B^{0, s}}^{\frac{5}{4 \alpha}}+\|v\|_{B^{0, s}}\right)\left(\|v\|_{B^{0, s}}^{1-\frac{1}{\alpha}}\left\|\nabla_{h}^{\alpha} v\right\|_{B^{0, s}}^{\frac{1}{\alpha}}+\|v\|_{B^{0, s}}\right) .
\end{aligned}
$$

Thus we conclude the proof of (2.5).

If $v=u$, by Hölder inequality, we infer that

$$
\left(F_{k}^{h, 1} \mid \Delta_{k}^{v} u\right)_{L^{2}} \leq \sum_{\left|k-k^{\prime}\right| \leq N_{0}}\left\|\Delta_{k}\left(S_{k^{\prime}-1}^{v} u^{h} \cdot \nabla_{h} \Delta_{k^{\prime}}^{v} u\right)\right\|_{L_{v}^{2}\left(L_{h}^{\frac{4}{3}}\right)}\left\|\Delta_{k}^{v} u\right\|_{L_{v}^{2}\left(L_{h}^{4}\right)} .
$$

By Proposition 2, we can deduce that

$$
\left\|\Delta_{k}^{v} u\right\|_{L_{v}^{2}\left(L_{h}^{4}\right)} \leq d_{k} 2^{-k s}\|u\|_{B^{0, s}}^{\frac{1}{2}}\left\|\nabla_{h} u\right\|_{B^{0, s}}^{\frac{1}{2}} .
$$

Using Bernstein inequality and Hölder inequality, we get

$$
\begin{aligned}
& \left\|\Delta_{k}^{v}\left(S_{k^{\prime}-1} u^{h} \cdot \nabla_{h} \Delta_{k^{\prime}}^{v} u\right)\right\|_{L_{v}^{2}\left(L_{h}^{\frac{4}{3}}\right)} \leq C\left\|S_{k^{\prime}-1}^{v} u^{h}\right\|_{L_{v}^{\infty}\left(L_{h}^{4}\right)}\left\|\nabla_{h} \Delta_{k^{\prime}}^{v} u\right\|_{L_{v}^{2}\left(L_{h}^{2}\right)} \\
& \quad \leq C 2^{-k^{\prime} s} d_{k^{\prime}}\left\|u^{h}\right\|_{L_{v}^{\infty}\left(L_{h}^{4}\right)}\left\|\nabla_{h} u\right\|_{B^{0, s}} \\
& \quad \leq C 2^{-k^{\prime} s} d_{k^{\prime}}\|u\|_{L_{v}^{\infty}\left(L_{h}^{2}\right)}^{\frac{1}{2}}\left\|\nabla_{h} u\right\|_{L_{v}^{\infty}\left(L_{h}^{2}\right)}^{\frac{1}{2}}\left\|\nabla_{h} u\right\|_{B^{0, s}} .
\end{aligned}
$$

Making use of (2.18) and (2.19) in (2.17), one can obtain that

$$
\left(F_{k}^{h, 1} \mid \Delta_{k}^{v} u\right)_{L^{2}} \leq C d_{k}^{2} 2^{-2 k s}\|u\|_{L_{v}^{\infty}\left(L_{h}^{2}\right)}^{\frac{1}{2}}\left\|\nabla_{h} u\right\|_{L_{v}^{\infty}\left(L_{h}^{2}\right)}^{\frac{1}{2}}\|u\|_{B^{0, s}}^{\frac{1}{2}}\left\|\nabla_{h} u\right\|_{B^{0, s}}^{\frac{3}{2}} .
$$

Arguing as in the estimate of term $F_{k}^{h, 1}$, we get

$$
\begin{aligned}
\left(F_{k}^{h, 2} \mid \Delta_{k}^{v} u\right)_{L^{2}} & \leq \sum_{\left|k-k^{\prime}\right| \leq N_{0}}\left\|\Delta_{k}^{v}\left(\Delta_{k^{\prime}}^{v} u^{h} \cdot \nabla_{h} S_{k^{\prime}-1}^{v} u\right)\right\|_{L_{v}^{2}\left(L_{h}^{\frac{4}{3}}\right)}\left\|\Delta_{k}^{v} u\right\|_{L_{v}^{2}\left(L_{h}^{4}\right)} \\
& \leq C \sum_{\left|k-k^{\prime}\right| \leq N_{0}}\left\|\Delta_{k^{\prime}}^{v} u\right\|_{L_{v}^{2}\left(L_{h}^{4}\right)}\left\|\nabla_{h} u\right\|_{L_{v}^{\infty}\left(L_{h}^{2}\right)}\left\|\Delta_{k}^{v} u\right\|_{L_{v}^{2}\left(L_{h}^{4}\right)} \\
& \leq C 2^{-2 k s} d_{k}^{2}\left\|\nabla_{h} u\right\|_{L_{v}^{\infty}\left(L_{h}^{2}\right)}\|u\|_{B^{0, s}}\left\|\nabla_{h} u\right\|_{B^{0, s}} .
\end{aligned}
$$

Similarly, for the term $F_{k}^{h, 3}$, we have

$$
\begin{aligned}
\left(F_{k}^{h, 3} \mid \Delta_{k}^{v} u\right)_{L^{2}} & \leq C \sum_{k^{\prime} \geq k-N_{0}}\left\|\Delta_{k^{\prime}}^{v} u^{h}\right\|_{L_{v}^{\infty}\left(L_{h}^{4}\right)}\left\|\widetilde{\Delta}_{k^{\prime}}^{v} \nabla_{h} u\right\|_{L_{v}^{2}\left(L_{h}^{2}\right)}\left\|\Delta_{k}^{v} u\right\|_{L_{v}^{2}\left(L_{h}^{4}\right)} \\
& \leq C \sum_{k^{\prime} \geq k-N_{0}}\|u\|_{L_{v}^{\infty}\left(L_{h}^{4}\right)}\left\|\widetilde{\Delta}_{k^{\prime}}^{v} \nabla_{h} u\right\|_{L^{2}}\|u\|_{B^{0, s}}^{\frac{1}{2}}\left\|\nabla_{h} u\right\|_{B^{0, s}}^{\frac{1}{2}}
\end{aligned}
$$




$$
\begin{aligned}
& \leq C d_{k}^{2} 2^{-2 k s}\|u\|_{L_{v}^{\infty}\left(L_{h}^{4}\right)}\|u\|_{B^{0, s}}^{\frac{1}{2}}\left\|\nabla_{h} u\right\|_{B^{0, s}}^{\frac{1}{2}} \sum_{k^{\prime} \geq k-N_{0}} 2^{\left(k-k^{\prime}\right) s} 2^{k^{\prime} s}\left\|\widetilde{\Delta}_{k^{\prime}}^{v} \nabla_{h} u\right\|_{L^{2}} \\
& \leq C d_{k}^{2} 2^{-2 k s}\|u\|_{L_{v}^{\infty}\left(L_{h}^{2}\right)}^{\frac{1}{2}}\left\|\nabla_{h} u\right\|_{L_{v}^{\infty}\left(L_{h}^{2}\right)}^{\frac{1}{2}}\|u\|_{B^{0, s}}^{\frac{1}{2}}\left\|\nabla_{h} u\right\|_{B^{0, s}}^{\frac{3}{2}},
\end{aligned}
$$

where we have used $s \geq 0$ in the last inequality of (2.20). The vertical term $F_{k}^{v}$ is also more delicate. Using Bony decomposition, along the same line as we did in the proof of (2.5), one can write

$$
\Delta_{k}^{v}\left(u^{3} \partial_{3} u\right)=F_{k}^{v, 1}+F_{k}^{v, 2}+F_{k}^{v, 3}
$$

with

$$
\begin{aligned}
F_{k}^{v, 1} & =\Delta_{k}^{v} \sum_{\left|k^{\prime}-k\right| \leq N_{0}} S_{k^{\prime}-1}^{v}\left(\partial_{3} u\right) \Delta_{k^{\prime}}^{v} u^{3}, \quad F_{k}^{v, 2}=\Delta_{k}^{v} \sum_{\left|k^{\prime}-k\right| \leq N_{0}} S_{k^{\prime}-1}^{v} u^{3} \partial_{3} \Delta_{k^{\prime}}^{v} u \\
F_{k}^{v, 3} & =\Delta_{k}^{v} \sum_{k^{\prime} \geq k-N_{0}} \widetilde{\Delta}_{k^{\prime}}^{v} u^{3} \partial_{3} \Delta_{k^{\prime}}^{v} u .
\end{aligned}
$$

For $F_{k}^{v, 1}$, we easily get that

$$
\begin{aligned}
\left(F_{k}^{v, 1} \mid \Delta_{k}^{v} u\right)_{L^{2}} & \leq C \sum_{\left|k^{\prime}-k\right| \leq N_{0}}\left\|S_{k^{\prime}-1}^{v}\left(\partial_{3} u\right) \Delta_{k^{\prime}}^{v} u^{3}\right\|_{L_{v}^{2}\left(L_{h}^{\frac{4}{3}}\right)}\left\|\Delta_{k}^{v} u\right\|_{L_{v}^{2}\left(L_{h}^{4}\right)} \\
& \leq C \sum_{\left|k^{\prime}-k\right| \leq N_{0}}\left\|\Delta_{k^{\prime}}^{v} u^{3}\right\|_{L_{v}^{2}\left(L_{h}^{2}\right)}\left\|\partial_{3} S_{k^{\prime}-1}^{v} u\right\|_{L_{v}^{\infty}\left(L_{h}^{4}\right)}\left\|\Delta_{k}^{v} u\right\|_{L_{v}^{2}\left(L_{h}^{4}\right)} \\
& \leq C \sum_{\left|k^{\prime}-k\right| \leq N_{0}}\left\|\Delta_{k^{\prime}}^{v} \partial_{3} u^{3}\right\|_{L_{v}^{2}\left(L_{h}^{2}\right)}\|u\|_{L_{v}^{\infty}\left(L_{h}^{4}\right)}\left\|\Delta_{k}^{v} u\right\|_{L_{v}^{2}\left(L_{h}^{4}\right)} .
\end{aligned}
$$

Proposition 2 implies that

$$
\left(F_{k}^{v, 1} \mid \Delta_{k}^{v} u\right)_{L^{2}} \leq C d_{k}^{2} 2^{-2 k s}\|u\|_{L_{v}^{\infty}\left(L_{h}^{2}\right)}^{\frac{1}{2}}\left\|\nabla_{h} u\right\|_{L_{v}^{\infty}\left(L_{h}^{2}\right)}^{\frac{1}{2}}\|u\|_{B^{0, s}}^{\frac{1}{2}}\left\|\nabla_{h} u\right\|_{B^{0, s}}^{\frac{3}{2}} .
$$

By the same argument as $F_{k}^{v, 1}$, we get

$$
\begin{aligned}
\left(F_{k}^{v, 3} \mid \Delta_{k}^{v} u\right)_{L^{2}} \leq & C \sum_{k^{\prime}>k-N_{0}}\left\|\widetilde{\Delta}_{k^{\prime}}^{v}\left(\partial_{3} u\right) \Delta_{k^{\prime}}^{v} u^{3}\right\|_{L_{v}^{2}\left(L_{h}^{\frac{4}{3}}\right)}\left\|\Delta_{k}^{v} u\right\|_{L_{v}^{2}\left(L_{h}^{4}\right)} \\
\leq & C \sum_{k_{k^{\prime}>k-N_{0}}}\left\|\Delta_{k^{\prime}}^{v} u^{3}\right\|_{L_{v}^{2}\left(L_{h}^{2}\right)}\left\|\partial_{3} \widetilde{\Delta}_{k^{\prime}}^{v} u\right\|_{L_{v}^{\infty}\left(L_{h}^{4}\right)}\left\|\Delta_{k}^{v} u\right\|_{L_{v}^{2}\left(L_{h}^{4}\right)} \\
\leq & C \sum_{\substack{k^{\prime}>k-N_{0} \\
k^{\prime} \geq 0}}\left\|\Delta_{k^{\prime}}^{v} \partial_{3} u^{3}\right\|_{L^{2}}\|u\|_{L_{v}^{\infty}\left(L_{h}^{4}\right)}\left\|\Delta_{k}^{v} u\right\|_{L_{v}^{2}\left(L_{h}^{4}\right)} \\
& +C\left\|\Delta_{-1}^{v} u^{3}\right\|_{L^{2}}\|u\|_{L_{v}^{\infty}\left(L_{h}^{4}\right)}\left\|\Delta_{k}^{v} u\right\|_{L_{v}^{2}\left(L_{h}^{4}\right)}
\end{aligned}
$$

Here, we also use the fact that when $k^{\prime}=-1$, the number of $k$ is finite. Again, Proposition 2 implies that

$$
\begin{aligned}
\left(F_{k}^{v, 3} \mid \Delta_{k}^{v} u\right)_{L^{2}} \leq & C d_{k}^{2} 2^{-2 k s}\|u\|_{L_{v}^{\infty}\left(L_{h}^{2}\right)}^{\frac{1}{2}}\left\|\nabla_{h} u\right\|_{L_{v}^{\infty}\left(L_{h}^{2}\right)}^{\frac{1}{2}}\|u\|_{B^{0, s}}^{\frac{1}{2}}\left\|\nabla_{h} u\right\|_{B^{0, s}}^{\frac{3}{2}} \\
& +C d_{k}^{2} 2^{-2 k s}\|u\|_{L_{v}^{\infty}\left(L_{h}^{2}\right)}^{\frac{1}{2}}\left\|\nabla_{h} u\right\|_{L_{v}^{\infty}\left(L_{h}^{2}\right)}^{\frac{1}{2}}\|u\|_{B^{0, s}}^{\frac{3}{2}}\left\|\nabla_{h} u\right\|_{B^{0, s}}^{\frac{1}{2}} .
\end{aligned}
$$


For the term $F_{k}^{v, 2}$, following the same computation as in [4] (Lemma 2.1), we get that

$$
\left(F_{k}^{v, 2} \mid \Delta_{k}^{v} u\right)_{L^{2}}=\left(S_{k}^{v} u^{3} \partial_{3} \Delta_{k}^{v} u \mid \Delta_{k}^{v} u\right)_{L^{2}}+R_{k}(u, u)
$$

with $R_{k}(u, u)$ defined by

$$
\left.\sum_{\left|k-k^{\prime}\right| \leq N_{0}}\left(\left[\Delta_{k}^{v}, S_{k^{\prime}-1}^{v} u^{3}\right] \partial_{3} \Delta_{k^{\prime}}^{v} u \mid \Delta_{k}^{v} u\right)_{L^{2}}+\sum_{\left|k-k^{\prime}\right| \leq N_{0}}\left(S_{k}^{v}-S_{k^{\prime}-1}^{v}\right) u^{3} \partial_{3} \Delta_{k^{\prime}}^{v} u \mid \Delta_{k}^{v} u\right)_{L^{2}}
$$

Then, using an integration by parts, we infer that

$$
\left(S_{k}^{v} u^{3} \partial_{3} \Delta_{k}^{v} u \mid \Delta_{k}^{v} u\right)_{L^{2}}=-\frac{1}{2}\left(S_{k}^{v}\left(\partial_{3} u^{3}\right) \Delta_{k}^{v} u \mid \Delta_{k}^{v} u\right)_{L^{2}} .
$$

Thanks to the fact that $u$ is divergence free, using Proposition 2, we get

$$
\begin{aligned}
\left(S_{k}^{v} u^{3} \partial_{3} \Delta_{k}^{v} u \mid \Delta_{k}^{v} u\right)_{L^{2}} & \leq C\left\|\nabla_{h} u\right\|_{L_{v}^{\infty}\left(L_{h}^{2}\right)}\left\|\Delta_{k}^{v} u\right\|_{L_{v}^{2}\left(L_{h}^{4}\right)}^{2} \\
& \leq C 2^{-2 k s} d_{k}^{2}\left\|\nabla_{h} u\right\|_{L_{v}^{\infty}\left(L_{h}^{2}\right)}\|u\|_{B^{0, s}}\left\|\nabla_{h} u\right\|_{B^{0, s}} .
\end{aligned}
$$

By Hölder inequality, we have

$$
\begin{aligned}
& \sum_{\left|k-k^{\prime}\right| \leq N_{0}}\left(\left[\Delta_{k}^{v}, S_{k^{\prime}-1}^{v} u^{3}\right] \partial_{3} \Delta_{k^{\prime}}^{v} u \mid \Delta_{k}^{v} u\right)_{L^{2}} \\
& \quad \leq C \sum_{\left|k-k^{\prime}\right| \leq N_{0}}\left\|\left[\Delta_{k}^{v}, S_{k^{\prime}-1}^{v} u^{3}\right] \partial_{3} \Delta_{k^{\prime}}^{v} u\right\|_{L_{v}^{2}\left(L_{h}^{\frac{4}{3}}\right)}\left\|\Delta_{k}^{v} u\right\|_{L_{v}^{2}\left(L_{h}^{4}\right)}
\end{aligned}
$$

Using Bernstein inequality and Proposition 2, we infer that

$$
\begin{aligned}
& \sum_{\left|k-k^{\prime}\right| \leq N_{0}}\left(\left[\Delta_{k}^{v}, S_{k^{\prime}-1}^{v} u^{3}\right] \partial_{3} \Delta_{k^{\prime}}^{v} u \mid \Delta_{k}^{v} u\right)_{L^{2}} \\
& \quad \leq C \sum_{\left|k-k^{\prime}\right| \leq N_{0}} 2^{k^{\prime}-k}\left\|S_{k^{\prime}-1}^{v} \partial_{3} u^{3}\right\|_{L_{v}^{\infty}\left(L_{h}^{2}\right)}\left\|\Delta_{k^{\prime}}^{v} u\right\|_{L_{v}^{2}\left(L_{h}^{4}\right)}\left\|\Delta_{k}^{v} u\right\|_{L_{v}^{2}\left(L_{h}^{4}\right)} \\
& \quad \leq C\left\|\nabla_{h} u\right\|_{L_{v}^{\infty}\left(L_{h}^{2}\right)}\left\|\Delta_{k^{\prime}}^{v} u\right\|_{L_{v}^{2}\left(L_{h}^{4}\right)}\left\|\Delta_{k}^{v} u\right\|_{L_{v}^{2}\left(L_{h}^{4}\right)} \\
& \quad \leq C 2^{-2 k s} d_{k}^{2}\left\|\nabla_{h} u\right\|_{L_{v}^{\infty}\left(L_{h}^{2}\right)}\|u\|_{B^{0, s}}\left\|\nabla_{h} u\right\|_{B^{0, s}}
\end{aligned}
$$

Since $\left(S_{k}^{v}-S_{k^{\prime}-1}^{v}\right) u^{3}$ is localized in frequency in a ring of size $2^{q}$ in the vertical variable, we get

$$
\begin{aligned}
& \left(\left(S_{k}^{v}-S_{k^{\prime}-1}\right) u^{3} \partial_{3} \Delta_{k^{\prime}}^{v} u \mid \Delta_{k}^{v} u\right)_{L^{2}} \\
& \quad \leq C \sum_{\left|k-k^{\prime}\right| \leq N_{0}} 2^{k^{\prime}-k}\left\|\left(S_{k}^{v}-S_{k^{\prime}-1}^{v}\right) \partial_{3} u^{3}\right\|_{L_{v}^{\infty}\left(L_{h}^{2}\right)}\left\|\Delta_{k^{\prime}}^{v} u\right\|_{L_{v}^{2}\left(L_{h}^{4}\right)}\left\|\Delta_{k}^{v} u\right\|_{L_{v}^{2}\left(L_{h}^{4}\right)} \\
& \quad \leq C\left\|\nabla_{h} u\right\|_{L_{v}^{\infty}\left(L_{h}^{2}\right)}\left\|\Delta_{k^{\prime}}^{v} u\right\|_{L_{v}^{2}\left(L_{h}^{4}\right)}\left\|\Delta_{k}^{v} u\right\|_{L_{v}^{2}\left(L_{h}^{4}\right)} \\
& \quad \leq C 2^{-2 k s} d_{k}^{2}\left\|\nabla_{h} u\right\|_{L_{v}^{\infty}\left(L_{h}^{2}\right)}\|u\|_{B^{0, s}}\left\|\nabla_{h} u\right\|_{B^{0, s}}
\end{aligned}
$$

Combing (2.25) with (2.26), we finally obtain that

$$
R_{k}(u, u) \leq C 2^{-2 k s} d_{k}^{2}\left\|\nabla_{h} u\right\|_{L_{v}^{\infty}\left(L_{h}^{2}\right)}\|u\|_{B^{0, s}}\left\|\nabla_{h} u\right\|_{B^{0, s}} .
$$


Thus (2.21), (2.22), (2.24) and (2.27) imply that the estimate (2.6) holds true.

We will also use the so-called Chemin-Lerner type spaces $\widetilde{L}_{T}^{\rho}\left(B^{0, s}\right)$.

Definition 2. For $s \in \mathbb{R}$, and $T>0$, we set

$$
\|u\|_{\widetilde{L}_{T}^{\rho}\left(B^{0, s}\right)}=\sum_{q \geq-1} 2^{q s}\left(\int_{0}^{T}\left\|\Delta_{q}^{v} u(t)\right\|_{L^{p}}^{\rho} d t\right)^{\frac{1}{\rho}}
$$

and denote by $\widetilde{L}_{T}^{\rho}\left(B^{0, s}\right)$ the subset of distributions $u \in \mathcal{S}^{\prime}\left([0, T] \times \mathbb{R}^{3}\right)$ with finite $\|u\|_{\widetilde{L}_{T}^{\rho}\left(B^{0, s}\right)}$ norm. When $\rho=\infty$, we take $\operatorname{ess} \sup _{t \in[0, T]}\|\cdot\|$ instead of $\|\cdot\|_{L^{\rho}[0, T]} \cdot$

\section{Local Existence of the Solution}

The purpose of this section is to prove the following local wellposedness result:

Theorem 2. Let $\alpha \geq \frac{3}{2}, \beta=1, s \geq 0$, and $u_{0} \in B^{0, s}$ be a divergence free vector field. There exist a positive time $T$ and a unique solution $u$ of (1.1) defined on $[0, T] \times \mathbb{R}^{3}$ such that

$$
u \in C\left([0, T], B^{0, s}\right) \quad \text { and } \quad \nabla_{h}^{\alpha} u \in \widetilde{L}^{2}\left(0, T ; B^{0, s}\right), \quad \partial_{3} u \in \widetilde{L}^{2}\left(0, T ; B^{0, s}\right) .
$$

Moreover, if the maximal time $T^{*}$ of existence is finite, then

$$
\lim _{t \rightarrow T^{*}} \int_{0}^{t}\left(\|u\|_{L_{v}^{\infty}\left(L_{h}^{2}\right)}^{\frac{4 \alpha-2}{4 \alpha-3}}\left\|\nabla_{h}^{\alpha} u\right\|_{L_{v}^{\infty}\left(L_{h}^{2}\right)}^{\frac{2}{4 \alpha-3}}+\|u\|_{L_{v}^{\infty}\left(L_{h}^{2}\right)}^{\frac{2 \alpha-2}{2 \alpha-1}}\left\|\nabla_{h}^{\alpha} u\right\|_{L_{v}^{\infty}\left(L_{h}^{2}\right)}^{\frac{2}{2 \alpha-1}}\right) d \tau=\infty
$$

\subsection{Local existence result}

In order to prove the local existence of solutions to system (1.1) (Theorem 2), we shall use the classical Friderichs' approximate solutions to system (1.1) (for the details, see [1], Chapter 10). To this end, let us define the sequence of operators $\left(P_{n}\right)_{n \in \mathbb{N}}$ by

$$
P_{n} u:=\mathcal{F}^{-1}\left(1_{B_{(0, n)}} \hat{u}\right),
$$

and we define the following approximate system:

$$
\left\{\begin{array}{l}
\partial_{t} u_{n}+P_{n}\left(u_{n} \cdot \nabla u_{n}\right)+\nu_{h} D_{h}^{2 \alpha} u_{n}-\nu_{3} \partial_{3}^{2} u_{n}=-P_{n}\left(\nabla \Pi_{n}\right) \\
\operatorname{div} u^{n}=0 \\
\left.u^{n}\right|_{t=0}=u_{0}^{n}
\end{array}\right.
$$

where $\Pi_{n}$ is given by

$$
-(-\Delta)^{-1} \operatorname{div}\left(u_{n} \cdot \nabla u_{n}\right)
$$

Then Cauchy-Lipschitz Theorem gives a solution $u_{n \in \mathbb{N}}^{n}$ in $C^{1}\left(\left[0, T_{n}^{*}\right) ; L^{2}\right), T_{n}^{*}$ is the maximal lifespan of $u_{n}$. We aim to exhibit a positive lower bound $T$ of 
$T_{n}^{*}$ and prove the uniform estimates of $u_{n}$. For that, we introduce the solution $v$ to the heat equations, that is

$$
\left\{\begin{array}{l}
\partial_{t} v+\nu_{h} D_{h}^{2 \alpha} v-\nu_{3} \partial_{3}^{2} v=0 \\
\left.v\right|_{t=0}=S_{N} u_{0}
\end{array}\right.
$$

where $S_{N} f=\mathcal{F}^{-1}\left(\chi\left(2^{-N}|\xi|\right) \hat{f}\right)$ and $N$ will be determined later. Clearly (3.2) has a unique global solution satisfying

$$
v_{N} \in C\left([0, \infty) ; B^{0, s}\left(\mathbb{R}^{3}\right)\right), \quad \nabla_{h}^{\alpha} v_{N} \text { and } \partial_{3} v_{N} \in \widetilde{L}^{2}\left(0, \infty ; B^{0, s}\left(\mathbb{R}^{3}\right)\right) .
$$

We want to show that if $T$ is small enough, then $\widetilde{u}_{n}:=u_{n}-v_{n, N}$ is small with the related norm, where $v_{n, N}:=P_{n} v_{N}$. To this end, we shall discuss the following perturbation system:

$$
\left\{\begin{array}{l}
\partial_{t} \widetilde{u}_{n}+D_{h}^{2 \alpha} \widetilde{u}_{n}-\partial_{3}^{2} \widetilde{u}_{n}+\nabla \Pi_{n}=H, \\
\operatorname{div} \widetilde{u}_{n}=0 \\
\left.\widetilde{u}_{n}\right|_{t=0}=\left(\operatorname{Id}-S_{N}\right) u_{0}^{n}
\end{array}\right.
$$

where

$$
H=-\left(\widetilde{u}_{n}+v_{n, N}\right) \cdot \nabla v_{n}-\left(\widetilde{u}_{n}+v_{n, N}\right) \cdot \nabla \widetilde{u}_{n} .
$$

We shall now be interested in proving that $\widetilde{u}_{n, N}$ exists on a uniform time interval. Applying $\Delta_{k}^{v}$ to (3.3) and then taking the $L^{2}$ inner product of the resulting equation with $\Delta_{k}^{v} \widetilde{u}_{n, N}$, we infer that

$$
\frac{d}{d t}\left\|\Delta_{k}^{v} \widetilde{u}_{n}\right\|_{L^{2}}^{2}+2 \nu_{h}\left\|\nabla_{h}^{\alpha} \Delta_{k}^{v} \widetilde{u}_{n}\right\|_{L^{2}}^{2}+2 \nu_{3}\left\|\partial_{3} \Delta_{k}^{v} \widetilde{u}_{n}\right\|_{L^{2}}^{2} \leq C\left|\left\langle\Delta_{k}^{v} H, \Delta_{k}^{v} \widetilde{u}_{n}\right\rangle\right| .
$$

Performing a time integration, multiplying both sides of (3.4) with $2^{k s}$ and summing on $k$, we obtain that

$$
\begin{aligned}
& \left\|\widetilde{u}_{n}\right\|_{B^{0, s}}+\sqrt{\nu_{h}}\left\|\nabla_{h}^{\alpha} \widetilde{u}_{n}\right\|_{\widetilde{L}_{t}^{2}\left(B^{0, s}\right)}+\sqrt{\nu_{3}}\left\|\partial_{3} \widetilde{u}_{n}\right\|_{\widetilde{L}_{t}^{2}\left(B^{0, s}\right)} \\
& \leq\left\|\widetilde{u}_{n}(0)\right\|_{B^{0, s}}+C \sum_{k \geq-1}\left(2^{2 k s} \int_{0}^{t}\left|\left(\Delta_{k}^{v} H \mid \Delta_{k}^{v} \widetilde{u}_{n}\right)_{L^{2}}\right| d \tau\right)^{\frac{1}{2}} .
\end{aligned}
$$

By Lemma 2.4 and the definition of $H$, one has

$$
\begin{aligned}
& \left|\left(\Delta_{k}^{v} H \mid \Delta_{k}^{v} \widetilde{u}_{n}\right)_{L^{2}}\right| \leq\left|\left(\Delta_{k}^{v}\left(\widetilde{u}_{n} \cdot \nabla \widetilde{u}_{n}\right) \mid \Delta_{k}^{v} \widetilde{u}_{n}\right)_{L^{2}}\right|+\left|\left(\Delta_{k}^{v}\left(v_{n, N} \cdot \nabla \widetilde{u}_{n}\right) \mid \Delta_{k}^{v} \widetilde{u}_{n}\right)_{L^{2}}\right| \\
& \quad+\left|\left(\Delta_{k}^{v}\left(\widetilde{u}_{n} \cdot \nabla v_{n, N}\right) \mid \Delta_{k}^{v} \widetilde{u}_{n}\right)_{L^{2}}\right|+\left|\left(\Delta_{k}^{v}\left(v_{n, N} \cdot \nabla v_{n, N}\right) \mid \Delta_{k}^{v} \widetilde{u}_{n}\right)_{L^{2}}\right| \cdot
\end{aligned}
$$

For the first two terms in the right hand side of (3.5), we apply Lemma 1 to obtain that

$$
\begin{aligned}
& \left|\left(\Delta_{k}^{v}\left(\widetilde{u}_{n} \cdot \nabla \widetilde{u}_{n}\right) \mid \Delta_{k}^{v} \widetilde{u}_{n}\right)_{L^{2}}\right| \\
& \leq d_{k}^{2} 2^{-2 k s}\left\|\widetilde{u}_{n}\right\|_{L_{v}^{\infty}\left(L_{h}^{2}\right)}\left(\left\|\widetilde{u}_{n}\right\|_{B^{0, s}}^{1-\frac{5}{4 \alpha}}\left\|\nabla_{h}^{\alpha} \widetilde{u}_{n}\right\|_{B^{0, s}}^{\frac{5}{4 \alpha}}+\left\|\widetilde{u}_{n}\right\|_{B^{0, s}}\right) \\
& \quad \times\left(\left\|\widetilde{u}_{n}\right\|_{B^{0}, s}^{1-\frac{1}{\alpha}}\left\|\nabla_{h}^{\alpha} \widetilde{u}_{n}\right\|_{B^{0, s}}^{\frac{1}{\alpha}}+\left\|\widetilde{u}_{n}\right\|_{B^{0, s}}\right) \\
& \leq C_{s} d_{k}^{2} 2^{-2 k s}\left\|\widetilde{u}_{n}\right\|_{B^{0, s}}^{2}\left(\left\|\widetilde{u}_{n}\right\|_{B^{0, s}}^{\frac{8 \alpha}{6 \alpha-9}}+\left\|\widetilde{u}_{n}\right\|_{B^{0, s}}^{\frac{8 \alpha}{6 \alpha-5}}+\left\|\widetilde{u}_{n}\right\|_{B^{0, s}}^{\frac{4 \alpha}{3 \alpha-2}}+\left\|\widetilde{u}_{n}\right\|_{B^{0, s}}^{\frac{4}{3}}\right) \\
& \quad+d_{k}^{2} 2^{-2 k s}\left(\frac{\nu_{3}}{256}\left\|\partial_{3} \widetilde{u}_{n}\right\|_{B^{0, s}}^{2}+\frac{\nu_{h}}{256}\left\|\nabla_{h}^{\alpha} \widetilde{u}_{n}\right\|_{B^{0, s}}^{2}\right)
\end{aligned}
$$


for $\alpha>\frac{3}{2}$ and

$$
\begin{aligned}
\mid\left(\Delta_{k}^{v}\right. & \left.\left(v_{n, N} \cdot \nabla \widetilde{u}_{n}\right) \mid \Delta_{k}^{v} \widetilde{u}_{n}\right)_{L^{2}} \mid \leq C_{s} d_{k}^{2} 2^{-2 k s}\left\|v_{n, N}\right\|_{L_{v}^{\infty}\left(L_{h}^{2}\right)} \\
& \times\left(\left\|\widetilde{u}_{n}\right\|_{B^{0, s}}^{1-\frac{5}{4 \alpha}}\left\|\nabla_{h}^{\alpha} \widetilde{u}_{n}\right\|_{B^{0, s}}^{\frac{5}{4 \alpha}}+\left\|\widetilde{u}_{n}\right\|_{B^{0, s}}\right)\left(\left\|\widetilde{u}_{n}\right\|_{B^{0, s}}^{1-\frac{1}{\alpha}}\left\|\nabla_{h}^{\alpha} \widetilde{u}_{n}\right\|_{B^{0, s}}^{\frac{1}{\alpha}}+\left\|\widetilde{u}_{n}\right\|_{B^{0, s}}\right) \\
& +C_{s} d_{k}^{2} 2^{-2 k s}\left\|\widetilde{u}_{n}\right\|_{L_{v}^{\infty}\left(L_{h}^{2}\right)}\left(\left\|v_{n, N}\right\|_{B^{0, s}}^{1-\frac{5}{4 \alpha}}\left\|\nabla_{h}^{\alpha} v_{n, N}\right\|_{B^{0, s}}^{\frac{5}{4 \alpha}}+\left\|v_{n, N}\right\|_{B^{0, s}}\right) \\
& \times\left(\left\|\widetilde{u}_{n}\right\|_{B^{0, s}}^{1-\frac{1}{\alpha}}\left\|\nabla_{h}^{\alpha} \widetilde{u}_{n}\right\|_{B^{0, s}}^{\frac{1}{\alpha}}+\left\|\widetilde{u}_{n}\right\|_{B^{0, s}}\right) \\
\leq & C_{s} d_{k}^{2} 2^{-2 k s}\left\|\widetilde{u}_{n}\right\|_{B^{0, s}}^{2}\left(\left\|v_{n, N}\right\|_{B^{0, s}}^{\frac{4 \alpha}{8 \alpha-9}}\left\|\partial_{3} v_{n, N}\right\|_{B^{0, s}}^{\frac{4 \alpha}{8 \alpha-9}}\right. \\
& +\left\|v_{n, N}\right\|_{B^{0, s}}^{\frac{1}{2}}\left\|\partial_{3} v_{n, N}\right\|_{B^{0, s}}^{\frac{1}{2}}+\left\|v_{n, N}\right\|_{B^{0, s}}^{\frac{4 \alpha}{8 \alpha-5}}\left\|\partial_{3} v_{n, N}\right\|_{B^{0, s}}^{\frac{4 \alpha}{8 \alpha-5}} \\
& +\left\|v_{n, N}\right\|_{B^{0, s}}^{\frac{\alpha}{2 \alpha-1}}\left\|\partial_{3} v_{n, N}\right\|_{B^{0, s}}^{\frac{\alpha}{2 \alpha-1}}+\left\|v_{n, N}\right\|_{B^{0, s}}^{\frac{4 \alpha-5}{3 \alpha-2}}\left\|\nabla_{h}^{\alpha} v_{n, N}\right\|_{B^{0, s}}^{\frac{5}{3 \alpha-2}} \\
& \left.+\left\|v_{n, N}\right\|_{B^{0, s}}^{\frac{4 \alpha-5}{3 \alpha}}\left\|\nabla_{h}^{\alpha} v_{n, N}\right\|_{B^{0, s}}^{\frac{5}{3 \alpha}}+\left\|v_{n, N}\right\|_{B^{0, s}}^{\frac{4 \alpha}{3 \alpha-2}}+\left\|v_{n, N}\right\|_{B^{0, s}}^{\frac{4}{3}}\right) \\
& +C_{s} d_{k}^{2} 2^{-2 k s}\left(\frac{\nu_{h}}{256}\left\|\nabla_{h}^{\alpha} \widetilde{u}_{n}\right\|_{B^{0, s}}^{2}+\frac{\nu_{3}}{256}\left\|\partial_{3} \widetilde{u}_{n}\right\|_{B^{0, s}}^{2}\right),
\end{aligned}
$$

where the sequence $d_{k}$ satisfies that $\left\|d_{k}\right\|_{l^{1}} \leq 1$. For the third term, we write

$$
\begin{aligned}
\left(\Delta_{k}^{v}\left(\widetilde{u}_{n} \cdot \nabla v_{n, N}\right) \mid \Delta_{k}^{v} \widetilde{u}_{n}\right)_{L^{2}}= & \left(\Delta_{k}^{v}\left(\widetilde{u}_{n}^{h} \cdot \nabla_{h} v_{n, N}\right) \mid \Delta_{k}^{v} \widetilde{u}_{n}\right)_{L^{2}} \\
& +\left(\Delta_{k}^{v}\left(\widetilde{u}_{n}^{3} \partial_{3} v_{n, N}\right) \mid \Delta_{k}^{v} \widetilde{u}_{n}\right)_{L^{2}} .
\end{aligned}
$$

By the same method as in (2.7), (2.8), (2.9) and (3.7), we have the following estimate

$$
\begin{aligned}
\mid\left(\Delta_{k}^{v}\right. & \left.\left(\widetilde{u}_{n}^{h} \cdot \nabla_{h} v_{n, N}\right) \mid \Delta_{k}^{v} \widetilde{u}_{n}\right)_{L^{2}} \mid \\
\leq & C_{s} d_{k}^{2} 2^{-2 k s}\left\|v_{n, N}\right\|_{L_{v}^{\infty}\left(L_{h}^{2}\right)}\left(\left\|\widetilde{u}_{n}\right\|_{B^{0, s}}^{1-\frac{5}{4 \alpha}}\left\|\nabla_{h}^{\alpha} \widetilde{u}_{n}\right\|_{B^{0, s}}^{\frac{5}{4 \alpha}}+\left\|\widetilde{u}_{n}\right\|_{B^{0, s}}\right) \\
& \times\left(\left\|\widetilde{u}_{n}\right\|_{B^{0, s}}^{1-\frac{1}{\alpha}}\left\|\nabla_{h}^{\alpha} \widetilde{u}_{n}\right\|_{B^{0, s}}^{\frac{1}{\alpha}}+\left\|\widetilde{u}_{n}\right\|_{B^{0, s}}\right) \\
& +C_{s} d_{k}^{2} 2^{-2 k s}\left\|\widetilde{u}_{n}\right\|_{L_{v}^{\infty}\left(L_{h}^{2}\right)}\left(\left\|v_{n, N}\right\|_{B^{0, s}}^{1-\frac{5}{4 \alpha}}\left\|\nabla_{h}^{\alpha} v_{n, N}\right\|_{B^{0, s}}^{\frac{5}{4 \alpha}}+\left\|v_{n, N}\right\|_{B^{0, s}}\right) \\
& \times\left(\left\|\widetilde{u}_{n}\right\|_{B^{0, s}}^{1-\frac{1}{\alpha}}\left\|\nabla_{h}^{\alpha} \widetilde{u}_{n}\right\|_{B^{0, s}}^{\frac{1}{\alpha}}+\left\|\widetilde{u}_{n}\right\|_{B^{0, s}}\right) \\
\leq & C_{s} d_{k}^{2} 2^{-2 k s}\left\|\widetilde{u}_{n}\right\|_{B^{0, s}}^{2}\left(\left\|v_{n, N}\right\|_{B^{0, s}}^{\frac{4 \alpha}{8 \alpha-9}}\left\|\partial_{3} v_{n, N}\right\|_{B^{0, s}}^{\frac{4 \alpha}{8 \alpha-9}}+\left\|v_{n, N}\right\|_{B^{0, s}}^{\frac{1}{2}}\left\|\partial_{3} v_{n, N}\right\|_{B^{0, s}}^{\frac{1}{2}}\right. \\
& +\left\|v_{n, N}\right\|_{B^{0, s}}^{\frac{4 \alpha}{8 \alpha-5}}\left\|\partial_{3} v_{n, N}\right\|_{B^{0, s}}^{\frac{4 \alpha}{8 \alpha-5}}+\left\|v_{n, N}\right\|_{B^{0, s}}^{\frac{\alpha}{2 \alpha-1}}\left\|\partial_{3} v_{n, N}\right\|_{B^{0, s}}^{\frac{\alpha}{2 \alpha-1}} \\
& +\left\|v_{n, N}\right\|_{B^{0, s}}^{\frac{4 \alpha-5}{3 \alpha-2}}\left\|\nabla_{h}^{\alpha} v_{n, N}\right\|_{B^{0, s}}^{\frac{5}{3 \alpha-2}}+\left\|v_{n, N}\right\|_{B^{0, s}}^{\frac{4 \alpha-5}{3 \alpha}}\left\|\nabla_{h}^{\alpha} v_{n, N}\right\|_{B^{0, s}}^{\frac{5}{3 \alpha}}+\left\|v_{n, N}\right\|_{B^{0, s}}^{\frac{4 \alpha}{3 \alpha-2}} \\
& \left.+\left\|v_{n, N}\right\|_{B^{0, s}}^{\frac{4}{3}}\right)+C_{s} d_{k}^{2} 2^{-2 k s}\left(\frac{\nu_{h}}{256}\left\|\nabla_{h}^{\alpha} \widetilde{u}_{n}\right\|_{B^{0, s}}^{2}+\frac{\nu_{3}}{256}\left\|\partial_{3} \widetilde{u}_{n}\right\|_{B^{0, s}}^{2}\right) \cdot(3.9)
\end{aligned}
$$

The second term of (3.8) can be estimated by the same method as Lemma 1. More precisely, by the Bony decomposition, we have

$$
\begin{aligned}
& \Delta_{k}^{v}\left(\widetilde{u}_{n} \partial_{3} v_{n, N}\right)=\sum_{\left|k-k^{\prime}\right| \leq N_{0}} \Delta_{k}^{v}\left(S_{k^{\prime}-1}^{v} \partial_{3} v_{n, N} \Delta_{k^{\prime}}^{v} \widetilde{u}_{n}^{3}\right) \\
& \quad+\sum_{\left|k-k^{\prime}\right| \leq N_{0}} \Delta_{k}^{v}\left(S_{k^{\prime}-1}^{v} \widetilde{u}_{n}^{3} \Delta_{k^{\prime}}^{v} \partial_{3} v_{n, N}\right)+\sum_{k^{\prime}>k-N_{0}} \Delta_{k}^{v}\left(\Delta_{k^{\prime}}^{v} \widetilde{u}_{n}^{3} \widetilde{\Delta}_{k^{\prime}}^{v} \partial_{3} v_{n, N}\right) .
\end{aligned}
$$


By the Proposition 1 in anisotropic space, one gets

$$
\begin{aligned}
& \sum_{\left|k-k^{\prime}\right| \leq N_{0}}\left(\Delta_{k}^{v}\left(S_{k^{\prime}-1}^{v} \widetilde{u}_{n}^{3} \Delta_{k^{\prime}}^{v} \partial_{3} v_{n, N}\right) \mid \Delta_{k}^{v} \widetilde{u}_{n}\right)_{L^{2}} \\
& \quad \leq C \sum_{\left|k-k^{\prime}\right| \leq N_{0}}\left\|S_{k^{\prime}-1}^{v} \widetilde{u}_{n}^{3}\right\|_{L_{v}^{\infty}\left(L_{h}^{2}\right)}\left\|\Delta_{k^{\prime}}^{v} \partial_{3} v_{n, N}\right\|_{L_{v}^{2}\left(L_{h}^{\frac{8}{3}}\right)}\left\|\Delta_{k}^{v} \widetilde{u}_{n}\right\|_{L_{v}^{2}\left(L_{h}^{8}\right)}
\end{aligned}
$$

Since $v_{n, N}$ is localized in the $\xi_{3}$ direction, we can apply Bernstein inequality to obtain

$$
\left\|\Delta_{k^{\prime}}^{v} \partial_{3} v_{n, N}\right\|_{L_{v}^{2}\left(L_{h}^{\frac{8}{3}}\right)} \leq C 2^{N}\left\|\Delta_{k^{\prime}}^{v} v_{n, N}\right\|_{L_{v}^{2}\left(L_{h}^{\frac{8}{3}}\right)} .
$$

Thus, interpolation and Young's inequalities imply that

$$
\begin{aligned}
& \sum_{\left|k-k^{\prime}\right| \leq N_{0}}\left(\Delta_{k}^{v}\left(S_{k^{\prime}-1}^{v} \widetilde{u}_{n}^{3} \Delta_{k^{\prime}}^{v} \partial_{3} v_{n, N}\right) \mid \Delta_{k}^{v} \widetilde{u}_{n}\right)_{L^{2}} \\
& \leq C 2^{N}\left\|\widetilde{u}_{n}\right\|_{L_{v}^{\infty}\left(L_{h}^{2}\right)} \sum_{\left|k^{\prime}-k\right| \leq N_{0}}\left\|\Delta_{k^{\prime}}^{v} v_{n, N}\right\|_{L^{2}}^{1-\frac{1}{4 \alpha}}\left\|\Delta_{k^{\prime}}^{v} \nabla_{h}^{\alpha} v_{n, N}\right\|_{L^{2}}^{\frac{1}{4 \alpha}} \\
& \quad \times\left\|\Delta_{k}^{v} \widetilde{u}_{n}\right\|_{L^{2}}^{1-\frac{3}{4 \alpha}}\left\|\Delta_{k}^{v} \nabla_{h}^{\alpha} \widetilde{u}_{n}\right\|_{L^{2}}^{\frac{3}{4 \alpha}} \\
& \leq C d_{k}^{2} 2^{-2 k s} 2^{N}\left\|\widetilde{u}_{n}\right\|_{B^{0, s}}^{\frac{1}{2}}\left\|\partial_{3} \widetilde{u}_{n}\right\|_{B^{0, s}}^{\frac{1}{2}}\left\|v_{n, N}\right\|_{B^{0, s}}^{1-\frac{1}{4 \alpha}}\left\|\nabla_{h}^{\alpha} v_{n, N}\right\|_{B^{0, s}}^{\frac{1}{4 \alpha}} \\
& \quad \times\left(\left\|\widetilde{u}_{n}\right\|_{B^{0, s}}^{1-\frac{3}{4 \alpha}}\left\|\nabla_{h}^{\alpha} \widetilde{u}_{n}\right\|_{B^{0, s}}^{\frac{3}{4 \alpha}}\right) \\
& \leq C d_{k}^{2} 2^{-2 k s} 2^{\frac{8 N \alpha}{6 \alpha-3}}\left\|\widetilde{u}_{n}\right\|_{B^{0, s}}^{2}\left\|v_{n, N}\right\|_{B^{0, s}}^{\frac{8 \alpha-2}{6 \alpha-3}}\left\|\nabla_{h}^{\alpha} v_{n, N}\right\|_{B^{0, s}}^{\frac{2}{6 \alpha-3}} \\
&+C_{s} d_{k}^{2} 2^{-2 k s}\left(\frac{\nu_{h}}{256}\left\|\nabla_{h}^{\alpha} \widetilde{u}_{n}\right\|_{B^{0, s}}^{2}+\frac{\nu_{3}}{256}\left\|\partial_{3} \widetilde{u}_{n}\right\|_{B^{0, s}}^{2}\right) .
\end{aligned}
$$

Similarly, we also have

$$
\begin{aligned}
& \sum_{\left|k-k^{\prime}\right| \leq N_{0}}\left(\Delta_{k}^{v}\left(S_{k^{\prime}-1}^{v} \partial_{3} v_{n, N} \Delta_{k^{\prime}}^{v} \widetilde{u}_{n, N}^{3}\right) \mid \Delta_{k}^{v} \widetilde{u}_{n}\right)_{L^{2}} \\
\leq & C 2^{N}\left\|v_{n, N}\right\|_{L_{v}^{\infty}\left(L_{h}^{2}\right)} \sum_{\left|k^{\prime}-k\right| \leq N_{0}}\left\|\Delta_{k^{\prime}}^{v} \widetilde{u}_{n}^{3}\right\|_{L^{2}}^{1-\frac{1}{4 \alpha}}\left\|\Delta_{k^{\prime}}^{v} \nabla_{h}^{\alpha} \widetilde{u}_{n}^{3}\right\|_{L^{2}}^{\frac{1}{4 \alpha}} \\
& \times\left\|\Delta_{k}^{v} \widetilde{u}_{n}\right\|_{L^{2}}^{1-\frac{3}{4 \alpha}}\left\|\Delta_{k}^{v} \nabla_{h}^{\alpha} \widetilde{u}_{n}\right\|_{L^{2}}^{\frac{3}{4 \alpha}} \\
\leq & C d_{k}^{2} 2^{-2 k s} 2^{N}\left\|v_{n, N}\right\|_{B^{0, s}}^{\frac{1}{2}}\left\|\partial_{3} v_{n, N}\right\|_{B^{0, s}}^{\frac{1}{2}}\left\|\widetilde{u}_{n}\right\|_{B^{0, s}}^{2-\frac{1}{\alpha}}\left\|\nabla_{h}^{\alpha} \widetilde{u}_{n}\right\|_{B^{0, s}}^{\frac{1}{\alpha}} \\
\leq & C d_{k}^{2} 2^{-2 k s} 2^{\frac{2 N \alpha}{2 \alpha-1}}\left\|\widetilde{u}_{n}\right\|_{B^{0, s}}^{2}\left\|v_{n, N}\right\|_{B^{0, s}}^{\frac{\alpha}{2 \alpha-1}}\left\|\nabla_{h}^{\alpha} v_{n, N}\right\|_{B^{0, s}}^{\frac{\alpha}{2 \alpha-1}} \\
& +C_{s} d_{k}^{2} 2^{-2 k s} \frac{\nu_{h}}{256}\left\|\nabla_{h}^{\alpha} \widetilde{u}_{n}\right\|_{B^{0, s}}^{2}
\end{aligned}
$$

and

$$
\begin{aligned}
* \sum_{k^{\prime}>k-N_{0}} & \left(\Delta_{k}^{v}\left(\Delta_{k^{\prime}}^{v} \widetilde{u}_{n}^{3} \widetilde{\Delta}_{k^{\prime}}^{v} \partial_{3} v_{n, N}\right) \mid \Delta_{k}^{v} \widetilde{u}_{n}\right)_{L^{2}} \\
\leq & C d_{k}^{2} 2^{-2 k s} 2^{\frac{8 N \alpha}{6 \alpha-3}}\left\|\widetilde{u}_{n}\right\|_{B^{0, s}}^{2}\left\|v_{n, N}\right\|_{B^{0, s}}^{\frac{8 \alpha-2}{6 \alpha-3}}\left\|\nabla_{h}^{\alpha} v_{n, N}\right\|_{B^{0, s}}^{\frac{2}{6 \alpha-3}} \\
& +C_{s} d_{k}^{2} 2^{-2 k s}\left(\frac{\nu_{h}}{256}\left\|\nabla_{h}^{\alpha} \widetilde{u}_{n}\right\|_{B^{0, s}}^{2}+\frac{\nu_{3}}{256}\left\|\partial_{3} \widetilde{u}_{n}\right\|_{B^{0, s}}^{2}\right) .
\end{aligned}
$$


What remains to estimate, therefore, is the term $\left(\Delta_{k}^{v}\left(v_{n, N} \cdot \nabla v_{n, N}\right) \mid \Delta_{k}^{v} \widetilde{u}_{n}\right)_{L^{2}}$. Since the vector field $v_{n, N}$ is divergence free, we can write

$$
\begin{aligned}
\left(\Delta_{k}^{v}\left(v_{n, N} \cdot \nabla v_{n, N}\right) \mid \Delta_{k}^{v} \widetilde{u}_{n}\right)_{L^{2}}= & -\left(\Delta_{k}^{v}\left(v_{n, N} \otimes v_{n, N}\right) \mid \Delta_{k}^{v} \nabla_{h} \widetilde{u}_{n}\right)_{L^{2}} \\
& +\left(\partial_{3} \Delta_{k}^{v}\left(v_{n, N} \otimes v_{n, N}\right) \mid \Delta_{k}^{v} \widetilde{u}_{n}\right)_{L^{2}} .
\end{aligned}
$$

Again, by Bony decomposition we have

$$
\begin{aligned}
\Delta_{k}^{v}\left(v_{n, N} \otimes v_{n, N}\right)= & 2 \sum_{\left|k-k^{\prime}\right| \leq N_{0}} \Delta_{k}^{v}\left(S_{k^{\prime}-1}^{v} v_{n, N} \otimes \Delta_{k^{\prime}}^{v} v_{n, N}\right) \\
& +\sum_{k^{\prime}>k-N_{0}} \Delta_{k}^{v}\left(\Delta_{k^{\prime}}^{v} v_{n, N} \otimes \widetilde{\Delta}_{k^{\prime}}^{v} v_{n, N}\right)
\end{aligned}
$$

Thus we have the estimate

$$
\begin{aligned}
& \sum_{\left|k-k^{\prime}\right| \leq N_{0}}\left(\Delta_{k}^{v}\left(S_{k^{\prime}-1}^{v} v_{n, N} \otimes \Delta_{k^{\prime}}^{v} v_{n, N}\right) \mid \Delta_{k}^{v} \nabla_{h} \widetilde{u}_{n}\right)_{L^{2}} \\
& \quad \leq C \sum_{\left|k-k^{\prime}\right| \leq N_{0}}\left\|S_{k^{\prime}-1}^{v} v_{n, N}\right\|_{L_{v}^{\infty}\left(L_{h}^{2}\right)}\left\|\Delta_{k^{\prime}}^{v} v_{n, N}\right\|_{L_{v}^{2}\left(L_{h}^{\frac{2}{\alpha-1}}\right)}\left\|\Delta_{k}^{v} \nabla_{h} \widetilde{u}_{n}\right\|_{L_{v}^{2}\left(L_{h}^{\frac{2}{2-\alpha}}\right)} \\
& \leq C\left\|v_{n, N}\right\|_{L_{v}^{\infty}\left(L_{h}^{2}\right)} \sum_{\left|k^{\prime}-k\right| \leq N_{0}}\left\|\Delta_{k^{\prime}}^{v} v\right\|_{L^{2}}^{2-\frac{2}{\alpha}}\left\|\Delta_{k^{\prime}}^{v} \nabla_{h}^{\alpha} v\right\|_{L^{2}}^{\frac{2}{\alpha}-1}\left\|\Delta_{k}^{v} \nabla_{h}^{\alpha} \widetilde{u}_{n}\right\|_{L^{2}} \\
& \leq C d_{k}^{2} 2^{-2 k s}\left\|v_{n, N}\right\|_{B^{0, s}}^{\frac{1}{2}}\left\|\partial_{3} v_{n, N}\right\|_{B^{0, s}}^{\frac{1}{2}}\left\|v_{n, N}\right\|_{B^{0, s}}^{2-\frac{2}{\alpha}}\left\|\nabla_{h}^{\alpha} v_{n, N}\right\|_{B^{0, s}}^{\frac{2}{\alpha}-1}\left\|\nabla_{h}^{\alpha} \widetilde{u}_{n}\right\|_{B^{0, s}} \\
& \leq C d_{k}^{2} 2^{-2 k s}\left(\left\|v_{n, N}\right\|_{B^{0, s}}^{5-\frac{4}{\alpha}}\left\|\partial_{3} v_{n, N}\right\|_{B^{0, s}}\left\|\nabla_{h}^{\alpha} v_{n, N}\right\|_{B^{0, s}}^{\frac{4}{\alpha}-2}+\frac{\nu_{h}}{256}\left\|\nabla_{h}^{\alpha} \widetilde{u}_{n}\right\|_{B^{0, s}}^{2}\right)
\end{aligned}
$$

and

$$
\begin{aligned}
& \sum_{k^{\prime}>k-N_{0}}\left(\Delta_{k}^{v}\left(\Delta_{k^{\prime}-1}^{v} v_{n, N} \otimes \widetilde{\Delta}_{k^{\prime}}^{v} v_{n, N}\right) \mid \Delta_{k}^{v} \nabla_{h} \widetilde{u}_{n}\right)_{L^{2}} \\
& \quad \leq C d_{k}^{2} 2^{-2 k s}\left(\left\|v_{n, N}\right\|_{B^{0, s}}^{5-\frac{4}{\alpha}}\left\|\partial_{3} v_{n, N}\right\|_{B^{0, s}}\left\|\nabla_{h}^{\alpha} v_{n, N}\right\|_{B^{0, s}}^{\frac{4}{\alpha}-2}+\frac{\nu_{h}}{256}\left\|\nabla_{h}^{\alpha} \widetilde{u}_{n}\right\|_{B^{0, s}}^{2}\right) .
\end{aligned}
$$

According the same method as in (3.10), (3.11) and the fact that $v_{n, N}$ is localized in the $\xi_{3}$ direction, one has

$$
\begin{aligned}
& \sum_{\left|k-k^{\prime}\right| \leq N_{0}}\left(\Delta_{k}^{v} \partial_{3}\left(S_{k^{\prime}-1}^{v} v_{n, N} \otimes \Delta_{k^{\prime}}^{v} v_{n, N}\right) \mid \Delta_{k}^{v} \widetilde{u}_{n}\right)_{L^{2}} \\
& \leq C 2^{N} \sum_{\left|k-k^{\prime}\right| \leq N_{0}}\left\|S_{k^{\prime}-1}^{v} v_{n, N}\right\|_{L_{v}^{\infty}\left(L_{h}^{2}\right)}\left\|\Delta_{k^{\prime}}^{v} v_{n, N}\right\|_{L_{v}^{2}\left(L_{h}^{\frac{8}{3}}\right)}\left\|\Delta_{k}^{v} \widetilde{u}_{n}\right\|_{L_{v}^{2}\left(L_{h}^{8}\right)} \\
& \leq C 2^{N}\left\|v_{n, N}\right\|_{L_{v}^{\infty}\left(L_{h}^{2}\right)} \sum_{\left|k^{\prime}-k\right| \leq N_{0}}\left\|\Delta_{k^{\prime}}^{v} v\right\|_{L^{2}}^{1-\frac{1}{4 \alpha}}\left\|\Delta_{k^{\prime}}^{v} \nabla_{h}^{\alpha} v\right\|_{L^{2}}^{\frac{1}{4 \alpha}} \\
& \quad \times\left\|\Delta_{k}^{v} \widetilde{u}_{n, N}\right\|_{L^{2}}^{1-\frac{3}{4 \alpha}}\left\|\Delta_{k}^{v} \nabla_{h}^{\alpha} \widetilde{u}_{n, N}\right\|_{L^{2}}^{\frac{3}{4 \alpha}}
\end{aligned}
$$




$$
\begin{aligned}
\leq & C d_{k}^{2} 2^{-2 k s} 2^{N}\left\|v_{n, N}\right\|_{B^{0, s}}^{\frac{1}{2}}\left\|\partial_{3} v_{n, N}\right\|_{B^{0, s}}^{\frac{1}{2}}\left\|v_{n, N}\right\|_{B^{0, s}}^{1-\frac{1}{4 \alpha}}\left\|\nabla_{h}^{\alpha} v_{n, N}\right\|_{B^{0, s}}^{\frac{1}{4 \alpha}} \\
& \times\left\|\widetilde{u}_{n}\right\|_{B^{0, s}}^{1-\frac{3}{4 \alpha}}\left\|\nabla_{h}^{\alpha} \widetilde{u}_{n}\right\|_{B^{0, s}}^{\frac{3}{4 \alpha}} \\
\leq & C_{N} d_{k}^{2} 2^{-2 k s}\left(\left\|v_{n, N}\right\|_{B^{0, s}}^{\frac{12 \alpha-2}{8 \alpha-3}}\left\|\partial_{3} v_{n, N}\right\|_{B^{0, s}}^{\frac{4 \alpha}{8 \alpha-3}}\left\|\nabla_{h}^{\alpha} v_{n, N}\right\| B_{B^{0, s}}^{\frac{2}{8 \alpha-3}}\left\|\widetilde{u}_{n}\right\|_{B^{0, s}}^{\frac{8 \alpha-6}{8 \alpha-3}}\right. \\
& \left.+\frac{\nu_{h}}{256}\left\|\nabla_{h}^{\alpha} \widetilde{u}_{n}\right\|_{B^{0, s}}^{2}\right)
\end{aligned}
$$

and

$$
\begin{aligned}
& \sum_{k^{\prime}>k-N_{0}}\left(\Delta_{k}^{v} \partial_{3}\left(\Delta_{k^{\prime}-1}^{v} v_{n, N} \otimes \widetilde{\Delta}_{k^{\prime}}^{v} v_{n, N}\right) \mid \Delta_{k}^{v} \widetilde{u}_{n}\right)_{L^{2}} \\
& \leq C_{N} d_{k}^{2} 2^{-2 k s}\left(\left\|v_{n, N}\right\|_{B^{0, s}}^{\frac{12 \alpha-2}{8 \alpha-3}}\left\|\partial_{3} v_{n, N}\right\|_{B^{0, s}}^{\frac{4 \alpha}{8 \alpha-3}}\left\|\nabla_{h}^{\alpha} v_{n, N}\right\|_{B^{0, s}}^{\frac{2}{8 \alpha-3}}\left\|\widetilde{u}_{n}\right\|_{B^{0, s}}^{\frac{8 \alpha-6}{8 \alpha-3}}\right. \\
& \left.\quad+\frac{\nu_{h}}{256}\left\|\nabla_{h}^{\alpha} \widetilde{u}_{n}\right\|_{B^{0, s}}^{2}\right) .
\end{aligned}
$$

To summarize, we combine above estimates (3.6)-(3.13) to obtain

$$
\begin{aligned}
&\left\|\widetilde{u}_{n}\right\|_{\widetilde{L}_{t}^{\infty}\left(B^{0, s}\right)}+\sqrt{\nu_{h}}\left\|\nabla_{h}^{\alpha} \widetilde{u}_{n}\right\|_{\widetilde{L}_{t}^{2}\left(B^{0, s}\right)}+\sqrt{\nu_{3}}\left\|\partial_{3} \widetilde{u}_{n}\right\|_{\widetilde{L}_{t}^{2}\left(B^{0, s}\right)} \\
& \leq\left\|\widetilde{u}_{n}(0)\right\|_{B^{0, s}}+C_{N}\left(\int _ { 0 } ^ { t } \| \widetilde { u } _ { n } \| _ { B ^ { 0 , s } } ^ { 2 } \left(\left\|\widetilde{u}_{n}\right\|_{B^{0, s}}^{\frac{8 \alpha}{6 \alpha-9}}+\left\|\widetilde{u}_{n}\right\|_{B^{0, s}}^{\frac{8 \alpha}{6 \alpha-5}}\right.\right. \\
&+\left\|\widetilde{u}_{n}\right\|_{B^{0, s}}^{\frac{4 \alpha}{3 \alpha-2}}+\left\|\widetilde{u}_{n}\right\|_{B^{0, s}}^{\frac{4}{3}}+\left\|v_{n, N}\right\|_{B^{0, s}}^{\frac{4 \alpha}{8 \alpha-9}}\left\|\partial_{3} v_{n, N}\right\|_{B^{0, s}}^{\frac{4 \alpha}{8 \alpha-9}} \\
&+\left\|v_{n, N}\right\|_{B^{0, s}}^{\frac{4 \alpha}{8 \alpha-5}}\left\|\partial_{3} v_{n, N}\right\|_{B^{0, s}}^{\frac{4 \alpha}{8 \alpha-5}}+\left\|v_{n, N}\right\|_{B^{0, s}}^{\frac{\alpha}{2 \alpha-1}}\left\|\partial_{3} v_{n, N}\right\|_{B^{0, s}}^{\frac{\alpha}{2 \alpha-1}} \\
&+\left\|v_{n, N}\right\|_{B^{0, s}}^{\frac{1}{2}}\left\|\partial_{3} v_{n, N}\right\|_{B^{0, s}}^{\frac{1}{2}}+\left\|v_{n, N}\right\|_{B^{0, s}}^{\frac{4 \alpha-5}{3 \alpha-2}}\left\|\nabla_{h}^{\alpha} v_{n, N}\right\|_{B^{0, s}}^{\frac{5}{3 \alpha-2}} \\
&+\left\|v_{n, N}\right\|_{B^{0, s}}^{\frac{4 \alpha-5}{3 \alpha}}\left\|\nabla_{h}^{\alpha} v_{n, N}\right\|_{B^{0, s}}^{\frac{5}{3 \alpha}}+\left\|v_{n, N}\right\|_{B^{0, s}}^{\frac{4 \alpha}{3 \alpha-2}}+\left\|v_{n, N}\right\|_{B^{0, s}}^{\frac{4}{3}} \\
&\left.+\left\|v_{n, N}\right\|_{B^{0, s}}^{\frac{8 \alpha-2}{6 \alpha-3}}\left\|\nabla_{h}^{\alpha} v_{n, N}\right\|_{B^{0, s}}^{\frac{2}{6 \alpha-3}}+\left\|v_{n, N}\right\|_{B^{0, s}}^{\frac{\alpha}{2 \alpha-1}}\left\|\nabla_{h}^{\alpha} v_{n, N}\right\|_{B^{0, s}}^{\frac{\alpha}{2 \alpha-1}}\right) \\
&+\left\|v_{n, N}\right\|_{B^{0, s}}^{5-\frac{4}{\alpha}}\left\|\partial_{3} v_{n, N}\right\|_{B^{0, s}}\left\|\nabla_{h}^{\alpha} v_{n, N}\right\|_{B^{0, s}}^{\frac{4}{\alpha}-2} \\
&\left.+\left\|\widetilde{u}_{n}\right\|_{B^{0, s}}^{\frac{8 \alpha-6}{8 \alpha-3}}\left\|v_{n, N}\right\|_{B^{0, s}}^{\frac{12 \alpha-2}{8 \alpha-3}}\left\|\partial_{3} v_{n, N}\right\|_{B^{0, s}}^{\frac{4 \alpha}{8 \alpha-3}}\left\|\nabla_{h}^{\alpha} v_{n, N}\right\|_{B^{0, s}}^{\frac{2}{8 \alpha-3}} d \tau\right)^{\frac{1}{2}} .
\end{aligned}
$$

Then we assume that for all $t \in[0, T]$

$$
\left\|\widetilde{u}_{n}(t)\right\|_{B^{0, s}} \leq 2 c .
$$

Here $T$ will be determined later. We want to prove that the above inequality holds strictly on $[0, T]$. Taking $N$ sufficiently large such that

$$
\left\|\left(\operatorname{Id}-S_{N}\right) u_{0}^{n}\right\|_{B^{0, s}} \leq c,
$$

then, for the fixed $N$, using the above estimate, one gets that

$$
\begin{aligned}
& \left\|\widetilde{u}_{n}\right\|_{\widetilde{L}_{t}^{\infty}\left(B^{0, s}\right)}+\sqrt{\nu_{h}}\left\|\nabla_{h}^{\alpha} \widetilde{u}_{n}\right\|_{\widetilde{L}_{t}^{2}\left(B^{0, s}\right)}+\sqrt{\nu_{3}}\left\|\partial_{3} \widetilde{u}_{n}\right\|_{\widetilde{L}_{t}^{2}\left(B^{0, s}\right)} \\
& \quad \leq c+C_{N}\left(4 c^{2} T+4 c^{2} T^{\frac{6 \alpha-9}{8 \alpha-9}}\left\|u_{0}\right\|_{B^{0, s}}^{4}+T^{\frac{3 \alpha-4}{2 \alpha}}\left\|u_{0}\right\|_{B^{0, s}}^{4}\right)^{\frac{1}{2}}
\end{aligned}
$$


for $\alpha>\frac{3}{2}$. While for $\alpha=\frac{3}{2}$, we just take the value of $\alpha$ in (3.6)-(3.13) except the estimate (3.6). Indeed, (3.6) can be rewritten as

$$
\begin{aligned}
\mid\left(\Delta_{k}^{v}\right. & \left.\left(\widetilde{u}_{n} \cdot \nabla \widetilde{u}_{n}\right) \mid \Delta_{k}^{v} \widetilde{u}_{n}\right)_{L^{2}} \mid \\
\leq & d_{k}^{2} 2^{-2 k s}\left\|\widetilde{u}_{n}\right\|_{L_{v}^{\infty}\left(L_{h}^{2}\right)}\left(\left\|\widetilde{u}_{n}\right\|_{B^{0, s}}^{\frac{1}{6}}\left\|\nabla_{h}^{\alpha} \widetilde{u}_{n}\right\|_{B^{0, s}}^{\frac{5}{6}}+\left\|\widetilde{u}_{n}\right\|_{B^{0, s}}\right) \\
& \times\left(\left\|\widetilde{u}_{n}\right\|_{B^{0, s}}^{\frac{1}{3}}\left\|\nabla_{h}^{\alpha} \widetilde{u}_{n}\right\|_{B^{0, s}}^{\frac{2}{3}}+\left\|\widetilde{u}_{n}\right\|_{B^{0, s}}\right) \\
\leq & C_{s} d_{k}^{2} 2^{-2 k s}\left\|\widetilde{u}_{n}\right\|_{B^{0, s}}^{2}\left(\left\|\widetilde{u}_{n}\right\|_{B^{0, s}}^{2}+\left\|\widetilde{u}_{n}\right\|_{B^{0, s}}^{3}+\left\|\widetilde{u}_{n}\right\|_{B^{0, s}}^{\frac{12}{5}}+\left\|\widetilde{u}_{n}\right\|_{B^{0, s}}^{\frac{4}{3}}\right) \\
& +d_{k}^{2} 2^{-2 k s}\left(\frac{\nu_{3}}{256}\left\|\partial_{3} \widetilde{u}_{n}\right\|_{B^{0, s}}^{2}+\frac{\nu_{h}}{256}\left\|\nabla_{h}^{\alpha} \widetilde{u}_{n}\right\|_{B^{0, s}}^{2}\right) .
\end{aligned}
$$

Thus we have

$$
\begin{aligned}
& \left\|\widetilde{u}_{n}\right\|_{\widetilde{L}_{t}^{\infty}\left(B^{0, s}\right)}+\sqrt{\nu_{h}}\left\|\nabla_{h}^{\alpha} \widetilde{u}_{n}\right\|_{\widetilde{L}_{t}^{2}\left(B^{0, s}\right)}+\sqrt{\nu_{3}}\left\|\partial_{3} \widetilde{u}_{n}\right\|_{\widetilde{L}_{t}^{2}\left(B^{0, s}\right)} \\
& \quad \leq c+C_{N}\left(4 c^{2} T+4 c^{2} T^{\frac{4}{9}}\left\|u_{0}\right\|_{B^{0, s}}^{4}+T^{\frac{1}{6}}\left\|u_{0}\right\|_{B^{0, s}}^{4}\right)^{\frac{1}{2}}
\end{aligned}
$$

for $\alpha=\frac{3}{2}$. Here, without loss generality, we assume that $T \leq 1, c \leq \frac{1}{4}$ and $\left\|u_{0}\right\|_{B^{0, s}} \geq 1$. For the fixed $c$, and $N$ which depends on $c$, we select a positive real number $T$ such that

$$
C_{N}\left(4 c^{2} T+4 c^{2} T^{\frac{6 \alpha-9}{8 \alpha-9}}\left\|u_{0}\right\|_{B^{0, s}}^{4}+T^{\frac{3 \alpha-4}{2 \alpha}}\left\|u_{0}\right\|_{B^{0, s}}^{4}\right)^{\frac{1}{2}} \leq \frac{1}{2} c .
$$

While for $\alpha=\frac{3}{2}$, we assume that

$$
C_{N}\left(4 c^{2} T+4 c^{2} T^{\frac{4}{9}}\left\|u_{0}\right\|_{B^{0, s}}^{4}+T^{\frac{1}{6}}\left\|u_{0}\right\|_{B^{0, s}}^{4}\right)^{\frac{1}{2}} \leq \frac{1}{2} c .
$$

Then we have

$$
\left\|\widetilde{u}_{n}(t)\right\|_{B^{0, s}} \leq \frac{3}{2} c, \quad \forall t \in[0, T] .
$$

This implies that $\widetilde{u}_{n}$ exists on a uniform time interval $[0, T], T$ is independent of $n$. A standard compactness argument which based on the Ascoli's theorem implies that $u^{n}$ tends to $u$ which satisfies (1.1). Moreover

$$
u \in \widetilde{L}^{\infty}\left(0, T ; B^{0, s}\right) \quad \text { and } \quad \nabla_{h}^{\alpha} u \in \widetilde{L}^{2}\left(0, T ; B^{0, s}\right), \quad \partial_{3} u \in \widetilde{L}^{2}\left(0, T ; B^{0, s}\right) .
$$

The continuity of the solution $u$ can be proved by the same method as [11] (Theorem 3.1). For the completeness, we give the details as follows. From (1.1), we have

$$
\Delta_{k}^{v} u_{t}=-\nu_{h} \Delta_{k}^{v} D_{h}^{\alpha} u+\nu_{3} \partial_{3}^{2} u-\Delta_{k}^{v}(u \cdot \nabla u)-\Delta_{k}^{v} \nabla \Pi .
$$

We can easily obtain that for all $k \geq-1$,

$$
\frac{d}{d t}\left\|\Delta_{k}^{v} u(t)\right\|_{L^{2}}^{2}=-\nu_{h}\left\|\nabla_{h}^{\alpha} \Delta_{k}^{v} u\right\|_{L^{2}}^{2}-\nu_{3}\left\|\partial_{3} \Delta_{k}^{v} u\right\|_{L^{2}}^{2}-\left(\Delta_{k}^{v}(u \cdot \nabla u) \mid \Delta_{k}^{v} u\right)_{L^{2}} .
$$


Obviously, we have

$$
\nu_{h}\left\|\nabla_{h}^{\alpha} \Delta_{k}^{v} u\right\|_{L^{2}}^{2}, \nu_{3}\left\|\partial_{3} \Delta_{k}^{v} u\right\|_{L^{2}}^{2} \in L^{1}([0, T]) .
$$

From Lemma 1, applying the method which we have used in (3.8), one can obtain that

$$
\left(\Delta_{k}^{v}(u \cdot \nabla u) \mid \Delta_{k}^{v} u\right)_{L^{2}} \in L^{1}([0, T])
$$

Then, we have $\frac{d}{d t}\left\|\Delta_{k}^{v} u(t)\right\|_{L^{2}}^{2} \in L^{1}([0, T])$ for all $k \geq-1$. Combining with the fact that $u \in \widetilde{L}^{\infty}\left(0, T ; B^{0, s}\right)$, we easily get that $u \in C\left([0, T] ; B^{0, s}\right)$.

\section{$3.2 \quad$ Uniqueness}

In this subsection, we will prove the uniqueness of the solution which obtained in the above subsection. For this end, we assume that $u_{1}$ and $u_{2}$ are the two solutions of (1.1) with the same initial data $u_{0}$, and

$u_{i} \in C\left([0, T] ; B^{0, s}\right), \quad \nabla_{h}^{\alpha} u_{i} \in L^{2}\left(0, T ; B^{0, s}\right), \quad \partial_{3} u_{i} \in L^{2}\left(0, T ; B^{0, s}\right), \quad i=1,2$.

Let $w:=u_{1}-u_{2}$, we have

$$
\partial_{t} w+\nu_{h} D_{h}^{2 \alpha} w-\nu_{3} \partial_{3}^{2} w+\nabla p=-w \cdot \nabla u_{2}-u_{1} \cdot \nabla w .
$$

Applying $\Delta_{k}^{v}$ to (3.14), taking the $L^{2}$ inner product of the resulting equation with $\Delta_{k}^{v} w$, then we infer that

$$
\begin{aligned}
& \frac{d}{d t}\left\|\Delta_{k}^{v} w\right\|_{L^{2}}^{2}+2 \nu_{h}\left\|\nabla_{h}^{\alpha} \Delta_{k}^{v} w\right\|_{L^{2}}^{2}+2 \nu_{3}\left\|\partial_{3} \Delta_{k}^{v} w\right\|_{L^{2}}^{2} \\
& \quad \leq 2\left|\left(\Delta_{k}^{v}\left(u_{1} \cdot \nabla w\right) \mid \Delta_{k}^{v} w\right)_{L^{2}}\right|+2\left|\left(\Delta_{k}^{v}\left(w \cdot \nabla u_{2}\right) \mid \Delta_{k}^{v} w\right)_{L^{2}}\right| .
\end{aligned}
$$

The first term of right side of (3.15) can be written as

$$
\left(\Delta_{k}^{v}\left(u_{1} \cdot \nabla w\right) \mid \Delta_{k}^{v} w\right)_{L^{2}}=\left(\Delta_{k}^{v}\left(u_{1}^{h} \cdot \nabla_{h} w\right) \mid \Delta_{k}^{v} w\right)_{L^{2}}+\left(\Delta_{k}^{v}\left(u_{1}^{3} \partial_{3} w\right) \mid \Delta_{k}^{v} w\right)_{L^{2}} .
$$

Applying the method as [3] (Lemma 1), we can easily get the bound of the right hand sides of (3.15). Indeed, for the first term of right side of (3.15), it can be written as

$$
\left(\Delta_{k}^{v}\left(u_{1} \cdot \nabla w\right) \mid \Delta_{k}^{v} w\right)_{L^{2}}=\left(\Delta_{k}^{v}\left(u_{1}^{h} \cdot \nabla_{h} w\right) \mid \Delta_{k}^{v} w\right)_{L^{2}}+\left(\Delta_{k}^{v}\left(u_{1}^{3} \partial_{3} w\right) \mid \Delta_{k}^{v} w\right)_{L^{2}} .
$$

We give the paraproduct algorithm of J. -M. Bony(see [2]), in the vertical which reads

$$
\begin{aligned}
\Delta_{k}^{v}\left(u_{1}^{h} \cdot \nabla_{h} w\right)= & \sum_{\left|k-k^{\prime}\right| \leq N_{0}} \Delta_{k}^{v}\left(S_{k^{\prime}-1}^{v} u_{1}^{h} \Delta_{k^{\prime}}^{v} w\right)+\sum_{\left|k-k^{\prime}\right| \leq N_{0}} \Delta_{k}^{v}\left(S_{k^{\prime}-1}^{v} w \Delta_{k^{\prime}}^{v} u_{1}\right) \\
& +\sum_{k^{\prime}>k-N_{0}} \Delta_{k}^{v}\left(\Delta_{k^{\prime}}^{v} u_{1} \widetilde{\Delta}_{k^{\prime}}^{v} w\right) .
\end{aligned}
$$


In this section, we just consider the case $s \in\left[\frac{1}{2}, \frac{3}{2}\right]$. For the case $s>\frac{3}{2}$, the uniqueness was proved in [3]. Thus by the praproduct law in 2-D Sobolev space and interpolation inequality, we have

$$
\begin{aligned}
& \left.\quad \sum_{\left|k-k^{\prime}\right| \leq \leq N_{0}} \Delta_{k}^{v}\left(S_{k^{\prime}-1}^{v} u_{1}^{h} \Delta_{k^{\prime}}^{v} \nabla_{h} w\right) \mid \Delta_{k}^{v} w\right)_{L^{2}} \\
& \quad \leq \sum_{\left|k-k^{\prime}\right| \leq N_{0}}\left\|S_{k^{\prime}-1}^{v} u_{1}\right\|_{L_{v}^{\infty}\left(L_{h}^{2}\right)}\left\|\Delta_{k^{\prime}}^{v} \nabla_{h} w\right\|_{L_{v}^{2}\left(\dot{H}^{\left.\frac{3}{4}\left(\mathbb{R}^{2}\right)\right)}\right.}\left\|\Delta_{k}^{v} w\right\|_{L_{v}^{2}\left(\dot{H}^{\frac{1}{4}}\left(\mathbb{R}^{2}\right)\right)} \\
& \leq C d_{k}^{2} 2^{-2 k(s-1)}\left\|u_{1}\right\|_{B^{0, s}}^{\frac{1}{2}}\left\|\partial_{3} u_{1}\right\|_{B^{0, s}}^{\frac{1}{2}}\|w\|_{B^{0, s-1}}^{2-\frac{2}{\alpha}}\left\|\nabla_{h}^{\alpha} w\right\|_{B^{0, s-1}}^{\frac{2}{\alpha}} \\
& \leq C d_{k}^{2} 2^{-2 k(s-1)}\left(C\left\|u_{1}\right\|_{B^{0, s}}^{\frac{\alpha}{2 \alpha-1}}\left\|\partial_{3} u_{1}\right\|_{B^{0, s-1}}^{\frac{\alpha}{2 \alpha-1}}\|w\|_{B^{0, s-1}}^{2}+\frac{\nu_{h}}{10}\left\|\nabla_{h}^{\alpha} w\right\|_{B^{0, s-1}}^{2}\right) .
\end{aligned}
$$

Similarly, according the same method, we also have

$$
\begin{aligned}
& \left.\sum_{\left|k-k^{\prime}\right| \leq N_{0}} \Delta_{k}^{v}\left(S_{k^{\prime}-1}^{v} \nabla_{h} w \Delta_{k^{\prime}}^{v} u_{1}^{h}\right) \mid \Delta_{k}^{v} w\right)_{L^{2}} \\
& \leq \sum_{\left|k-k^{\prime}\right| \leq N_{0}}\left\|S_{k^{\prime}-1}^{v} \nabla_{h} w\right\|_{L_{v}^{\infty}\left(\dot{H}^{\frac{3}{4}}\left(\mathbb{R}^{2}\right)\right)}\left\|\Delta_{k^{\prime}}^{v} u_{1}\right\|_{L^{2}}\left\|\Delta_{k}^{v} w\right\|_{L_{v}^{2}\left(\dot{H}^{\frac{1}{4}}\left(\mathbb{R}^{2}\right)\right)} \\
& \leq \sum_{\substack{\left|k-k^{\prime}\right| \leq N_{0} \\
k^{\prime \prime} \leq k^{\prime}-2}}\left\|\Delta_{k^{\prime \prime}}^{v} \nabla_{h} w\right\|_{L_{v}^{2}\left(\dot{H}^{\frac{3}{4}}\left(\mathbb{R}^{2}\right)\right)} 2^{k^{\prime \prime}(s-1)}\left\|\Delta_{k^{\prime}}^{v} u_{1}\right\|_{L^{2}} 2^{\frac{1}{2} k^{\prime}} \\
& \quad \times\left\|\Delta_{k}^{v} w\right\|_{L_{v}^{2}\left(\dot{H}^{\frac{1}{4}}\left(\mathbb{R}^{2}\right)\right)} 2^{\left(k^{\prime \prime}-k^{\prime}\right)\left(\frac{3}{2}-s\right)} 2^{-k^{\prime}(s-1)} \\
& \leq C d_{k}^{2} 2^{-2 k(s-1)}\left\|u_{1}\right\|_{B^{0, s}}^{\frac{1}{2}}\left\|\partial_{3} u_{1}\right\|_{B^{0, s}}^{\frac{1}{2}}\|w\|_{B^{0, s-1}}^{2-\frac{2}{\alpha}}\left\|\nabla_{h}^{\alpha} w\right\|_{B^{0, s-1}}^{\frac{2}{\alpha}} \\
& \leq C d_{k}^{2} 2^{-2 k(s-1)}\left(C\left\|u_{1}\right\|_{B^{0, s}}^{\frac{\alpha}{2 \alpha-1}}\left\|\partial_{3} u_{1}\right\|_{B^{0, s-1}}^{\frac{\alpha}{2 \alpha-1}}\|w\|_{B^{0, s-1}}^{2}+\frac{\nu_{h}}{10}\left\|\nabla_{h}^{\alpha} w\right\|_{B^{0, s-1}}^{2}\right)
\end{aligned}
$$

and

$$
\begin{aligned}
& \left.\sum_{k^{\prime}>k-N_{0}} \Delta_{k}^{v}\left(\Delta_{k^{\prime}}^{v} u_{1}^{h} \Delta_{k^{\prime}}^{v} \nabla_{h} w\right) \mid \Delta_{k}^{v} w\right)_{L^{2}} \leq \sum_{k^{\prime}>k-N_{0}}\left\|\Delta_{k^{\prime}}^{v} \nabla_{h} w\right\|_{L_{v}^{2}\left(\dot{H}^{\frac{3}{4}}\left(\mathbb{R}^{2}\right)\right)} 2^{k^{\prime}(s-1)} \\
& \quad \times\left\|\Delta_{k^{\prime}}^{v} u_{1}\right\|_{L^{2}} 2^{\frac{1}{2} k^{\prime}}\left\|\Delta_{k}^{v} w\right\|_{L_{v}^{2}\left(\dot{H}^{\left.\frac{1}{4}\left(\mathbb{R}^{2}\right)\right)}\right.} 2^{-k^{\prime}\left(s-\frac{1}{2}\right)} 2^{\frac{1}{2} k} \\
& \leq C d_{k}^{2} 2^{-2 k(s-1)}\left\|u_{1}\right\|_{B^{0, s}}^{\frac{1}{2}}\left\|\partial_{3} u_{1}\right\|_{B^{0, s}}^{\frac{1}{2}}\|w\|_{B^{0, s-1}}^{2-\frac{2}{\alpha}}\left\|\nabla_{h}^{\alpha} w\right\|_{B^{0, s-1}}^{\frac{2}{\alpha}} \\
& \leq C d_{k}^{2} 2^{-2 k(s-1)}\left(C\left\|u_{1}\right\|_{B^{0, s}}^{\frac{\alpha}{2 \alpha-1}}\left\|\partial_{3} u_{1}\right\|_{B^{0, s-1}}^{\frac{\alpha}{2 \alpha-1}}\|w\|_{B^{0, s-1}}^{2}+\frac{\nu_{h}}{10}\left\|\nabla_{h}^{\alpha} w\right\|_{B^{0, s-1}}^{2}\right) .
\end{aligned}
$$

In the last inequality, we have used the assumption that $s \geq \frac{1}{2}$. The same method used in the second term of the right hand sides of (3.15) implies that

$$
\begin{aligned}
\left(\Delta_{k}^{v}\left(w \cdot \nabla u_{2}\right) \mid \Delta_{k}^{v} w\right)_{L^{2}} & \\
\leq & C d_{k}^{2} 2^{-2 k(s-1)}\left\|\nabla_{h}^{\frac{1}{4}} w\right\|_{B^{0, s-1}}\left\|\nabla u_{2}\right\|_{B^{0, s}}\left\|\nabla_{h}^{\frac{3}{4}} w\right\|_{B^{0, s-1}} \\
\leq & d_{k}^{2} 2^{-2 k(s-1)}\left(C\left\|\partial_{3} u_{2}\right\|_{B^{0, s}}^{\frac{2 \alpha}{2 \alpha-1}}\|w\|_{B^{0, s-1}}^{2}+\frac{\nu_{h}}{10}\left\|\nabla_{h}^{\alpha} w\right\|_{B^{0, s-1}}^{2}\right) \\
& +C d_{k}^{2} 2^{-2 k(s-1)}\left\|u_{2}\right\|_{B^{0, s}}^{\frac{2 \alpha-2}{2 \alpha-1}}\left\|\nabla_{h}^{\alpha} u_{2}\right\|_{B^{0, s}}^{\frac{2}{2 \alpha-1}}\|w\|_{B^{0, s-1}}^{2} .
\end{aligned}
$$


To estimate the term $\left(\Delta_{k}^{v}\left(u_{1}^{3} \partial_{3} w\right) \mid \Delta_{k}^{v} w\right)_{L^{2}}$, let us again using Bony decomposition in the vertical variable:

$$
\Delta_{k}^{v}\left(u_{1}^{3} \partial_{3} w\right)=F_{k}^{v, 1}+F_{k}^{v, 2}
$$

with

$$
F_{k}^{v, 1}=\Delta_{k}^{v}\left(\sum_{k^{\prime} \geq k-N_{0}} S_{k^{\prime}+2}^{v}\left(\partial_{3} w\right) \Delta_{k^{\prime}}^{v} u_{1}^{3}\right), \quad F_{k}^{v, 2}=\Delta_{k}^{v}\left(\sum_{\left|k^{\prime}-k\right| \leq N_{0}} S_{k^{\prime}-1}^{v} u_{1}^{3} \partial_{3} \Delta_{k^{\prime}}^{v} w\right) .
$$

Bernstein's inequality, along with Proposition 1 yields

$$
\begin{aligned}
\left(F_{k}^{v, 1} \mid \Delta_{k}^{v} w\right)_{L^{2}} \leq & d_{k}^{2} 2^{-2 k(s-1)}\left(C\left\|\partial_{3} u_{1}\right\|_{B^{0, s}}^{\frac{\alpha}{2 \alpha-1}}\|w\|_{B^{0, s-1}}^{2}\right. \\
& \left.+\frac{\nu_{h}}{10}\left\|\nabla_{h}^{\alpha} w\right\|_{B^{0, s-1}}^{2}\right)\|w\|_{B^{0, s-1}}^{2},
\end{aligned}
$$

where $\sum_{k \geq-1} d_{k} \leq 1$. To get the estimate of the term $\left(F_{k}^{v, 2} \mid \Delta_{k}^{v} w\right)_{L^{2}\left(\mathbb{R}^{3}\right)}$, we have to use in a crucial way the structure of the nonlinearity. Following a computation done in [4], we get that

$$
\left(F_{k}^{v, 2} \mid \Delta_{k}^{v} w\right)_{L^{2}}=\left(S_{k}^{v} u_{1}^{3} \partial_{3} \Delta_{k}^{v} w \mid \Delta_{k}^{v} w\right)_{L^{2}}+R_{k}\left(u_{1}^{3}, w\right)
$$

with $R_{k}\left(u_{1}^{3}, w\right)$ defined by

$$
\begin{aligned}
& \sum_{\left|k-k^{\prime}\right| \leq N_{0}}\left(\left[\Delta_{k}^{v}, S_{k^{\prime}-2} u_{1}^{3}\right] \partial_{3} \Delta_{k^{\prime}}^{v} w \mid \Delta_{k}^{v} w\right)_{L^{2}} \\
& \left.+\sum_{\left|k-k^{\prime}\right| \leq N_{0}}\left(S_{k}^{v}-S_{k^{\prime}-2}^{v}\right) u_{1}^{3} \partial_{3} \Delta_{k^{\prime}}^{v} w \mid \Delta_{k}^{v} w\right)_{L^{2}}
\end{aligned}
$$

Then, using an integration by parts, we infer that

$$
\left(S_{k}^{v} u^{3} \partial_{3} \Delta_{k}^{v} w \mid \Delta_{k}^{v} w\right)_{L^{2}}=-\frac{1}{2}\left(S_{k}^{v}\left(\partial_{3} u_{1}^{3}\right) \Delta_{k}^{v} w \mid \Delta_{k}^{v} w\right)_{L^{2}} .
$$

So according the same method which discussed in Lemma1, we finally have

$$
\begin{aligned}
\left(\Delta_{k}^{v}\left(u_{1}^{3} \cdot \partial_{3} w\right) \mid \Delta_{k}^{v} w\right)_{L^{2}} \leq & C d_{k}^{2} 2^{-2 k(s-1)}\left(C\left\|u_{1}\right\|_{B^{0, s}}^{\frac{\alpha}{2 \alpha-1}}\left\|\partial_{3} u_{1}\right\|_{B^{0, s-1}}^{\frac{\alpha}{2 \alpha-1}}\|w\|_{B^{0, s-1}}^{2}\right. \\
& \left.+\frac{\nu_{h}}{10}\left\|\nabla_{h}^{\alpha} w\right\|_{B^{0, s-1}}^{2}\right)
\end{aligned}
$$

Integrating (3.15) over $[0, T]$, combining the above estimates (3.15)-(3.18), one can obtain that

$$
\begin{aligned}
& \|w(t)\|_{B^{0, s-1}}^{2}+\nu_{h}\left\|\nabla_{h}^{\alpha} w(t)\right\|_{\widetilde{L}_{t}^{2}\left(B^{0, s-1}\right)}^{2}+\nu_{3}\left\|\partial_{3} w\right\|_{\widetilde{L}_{t}^{2}\left(B^{0, s-1}\right)}^{2} d \tau \\
& \leq C \int_{0}^{t}\left(\left\|\partial_{3} u_{1}\right\|_{B^{0, s-1}}^{\frac{\alpha}{2 \alpha-1}}+\left\|\partial_{3} u_{2}\right\|_{B^{0, s-1}}^{\frac{2 \alpha}{2 \alpha-1}}\right)\|w\|_{B^{0, s-1}}^{2} d \tau \\
& \quad+C \int_{0}^{t}\left\|\nabla_{h}^{\alpha} u_{2}\right\|_{B^{0, s}}^{\frac{2}{2 \alpha-1}}\|w\|_{B^{0, s-1}}^{2} d \tau
\end{aligned}
$$

for all $t \in[0, T]$. Then using Gronwall's lemma to (3.16), we deduce that $w \equiv 0$ on $[0, \mathrm{~T}]$ and the uniqueness has been proved. 


\subsection{Blow-up criterion}

In this subsection, we will prove the blow-up criterion (3.1). First, let us denote by $T^{*}$ the maximal time of existence of system (1.1). Applying $\Delta_{k}^{v}$ to (1.1) and taking the $L^{2}$ inner product of the resulting equation with $\Delta_{k}^{v} u$, then performing a time integration, we can infer that

$$
\begin{gathered}
\|u\|_{L_{t}^{\infty}\left(B^{0, s}\right)}+\sqrt{\nu_{h}}\left\|\nabla_{h}^{\alpha} u\right\|_{L_{t}^{2}\left(B^{0, s}\right)}+\sqrt{\nu_{3}}\left\|\partial_{3} u\right\|_{L_{t}^{2}\left(B^{0, s}\right)} \\
\leq C \sum_{k \geq-1} 2^{k s}\left(\int_{0}^{t}\left|\left(\Delta_{k}^{v}(u \cdot \nabla u) \mid \Delta_{k}^{v} u\right)_{L^{2}}\right| d \tau\right)^{\frac{1}{2}}
\end{gathered}
$$

for all $t \in\left[0, T^{*}\right)$. Using Lemma 1 and interpolation inequality, we obtain

$$
\begin{aligned}
\left|\left(\Delta_{k}^{v}(u \cdot \nabla u) \mid \Delta_{k}^{v} u\right)_{L^{2}}\right| \leq & C d_{k} 2^{-2 k s}\left(\|u\|_{L_{v}^{\infty}\left(L_{h}^{2}\right)}^{\frac{1}{2}}\left\|\nabla_{h} u\right\|_{L_{v}^{\infty}\left(L_{h}^{2}\right)}^{\frac{1}{2}}\|u\|_{B^{0, s}}^{\frac{1}{2}}\left\|\nabla_{h} u\right\|_{B^{0, s}}^{\frac{3}{2}}\right. \\
& \left.+\left\|\nabla_{h} u\right\|_{L_{v}^{\infty}\left(L_{h}^{2}\right)}\|u\|_{B^{0, s}}\left\|\nabla_{h} u\right\|_{B^{0, s}}\right) \\
\leq & C d_{k}^{2} 2^{-2 k s}\left(\|u\|_{L_{v}^{\infty}\left(L_{h}^{2}\right)}^{1-\frac{1}{2 \alpha}}\left\|\nabla_{h}^{\alpha} u\right\|_{L_{v}^{\infty}\left(L_{h}^{2}\right)}^{\frac{1}{2 \alpha}}\|u\|_{B^{0, s}}^{2-\frac{3}{2 \alpha}}\left\|\nabla_{h}^{\alpha} u\right\|_{B^{0, s}}^{\frac{3}{2 \alpha}}\right. \\
& +\|u\|_{L_{v}^{\infty}\left(L_{h}^{2}\right)}^{1-\frac{1}{\alpha}}\left\|\nabla_{h}^{\alpha} u\right\|_{L_{v}^{\infty}\left(L_{h}^{2}\right)}^{\frac{1}{\alpha}}\|u\|_{B^{0, s}}^{2-\frac{1}{\alpha}}\left\|\nabla_{h}^{\alpha} u\right\|_{B^{0, s}}^{\frac{1}{\alpha}} \\
& \left.+\|u\|_{L_{v}^{\infty}\left(L_{h}^{2}\right)}^{12 \alpha}\left\|\nabla_{h}^{\alpha} u\right\|_{L_{v}^{\infty}\left(L_{h}^{2}\right)}^{\frac{1}{2 \alpha}}\|u\|_{B^{0, s}}^{2-\frac{1}{2 \alpha}}\left\|\nabla_{h}^{\alpha} u\right\|_{B^{0, s}}^{\frac{1}{2 \alpha}}\right) \\
\leq & d_{k}^{2} 2^{-2 k s} \frac{\nu_{h}}{2}\left\|\nabla_{h}^{\alpha} u\right\|_{B^{0, s}}^{2} \\
& +C d_{k}^{2} 2^{-2 k s} \nu_{h}^{-\frac{3}{4 \alpha-3}}\|u\|_{L_{v}^{\infty}\left(L_{h}^{2}\right)}^{\frac{4 \alpha-2}{4 \alpha-3}}\left\|\nabla_{h}^{\alpha} u\right\|_{L_{v}^{\infty}\left(L_{h}^{2}\right)}^{\frac{2}{4 \alpha-3}}\|u\|_{B^{0, s}}^{2} \\
& +C d_{k}^{2} 2^{-2 k s} \nu_{h}^{-\frac{1}{2 \alpha-1}}\|u\|_{L_{v}^{\infty}\left(L_{h}^{2}\right)}^{\frac{2 \alpha-2}{2 \alpha-1}}\left\|\nabla_{h}^{\alpha} u\right\|_{L_{v}^{\infty}\left(L_{h}^{2}\right)}^{\frac{2}{2 \alpha-1}}\|u\|_{B^{0, s}}^{2} \\
& +C d_{k}^{2} 2^{-2 k s} \nu_{h}^{-\frac{1}{4 \alpha-1}}\|u\|_{L_{v}^{\infty}\left(L_{h}^{2}\right)}^{\frac{4 \alpha-2}{4 \alpha-1}}\left\|\nabla_{h}^{\alpha} u\right\|_{L_{v}^{\infty}\left(L_{h}^{2}\right)}^{\frac{2}{4 \alpha-1}}\|u\|_{B^{0, s}}^{2} .
\end{aligned}
$$

The Gronwall's Lemma gives

$$
\begin{aligned}
\|u(t)\|_{B^{0, s}} \leq & \left\|u_{0}\right\|_{B^{0, s}} \exp \left\{\int _ { 0 } ^ { t } \left(\nu_{h}^{-\frac{3}{4 \alpha-3}}\|u(\tau)\|_{L_{v}^{\infty}\left(L_{h}^{2}\right)}^{\frac{4 \alpha-2}{4 \alpha-3}}\left\|\nabla_{h}^{\alpha} u\right\|_{L_{v}^{\infty}\left(L_{h}^{2}\right)}^{\frac{2}{4 \alpha-3}}\right.\right. \\
& +\nu_{h}^{-\frac{1}{4 \alpha-1}}\|u(\tau)\|_{L_{v}^{\infty}\left(L_{h}^{2}\right)}^{\frac{4 \alpha-2}{4 \alpha-1}}\left\|\nabla_{h}^{\alpha} u\right\|_{L_{v}^{\infty}\left(L_{h}^{2}\right)}^{\frac{2}{4 \alpha-1}} \\
& \left.\left.+\nu_{h}^{-\frac{1}{2 \alpha-1}}\|u\|_{L_{v}^{\infty}\left(L_{h}^{2}\right)}^{\frac{2 \alpha-2}{2 \alpha-1}}\left\|\nabla_{h}^{\alpha} u\right\|_{L_{v}^{\infty}\left(L_{h}^{2}\right)}^{\frac{2}{2 \alpha-1}}\right) d \tau\right\}
\end{aligned}
$$

Then if the right hand sides of (3.20) are bounded, then $T^{*}=\infty$. Otherwise, if $T^{*}$ is finite, we can define the value of $u\left(T^{*}\right)$ by $\lim _{t \rightarrow T^{*}} u(t)$ in $B^{0, s}$. Using $u\left(T^{*}\right)$ as a new initial data, applying the local existence result which have obtained before, we extend $u$ beyond $T^{*}$. This ends the proof of Theorem 2 .

\section{Global Solution of System (1.1)}

In order to show that the solution constructed in the previous section can be continued to the global one, we need the following a priori estimate. Note that 
in the present work, we assume that $u_{0} \in B^{0, s}$, for $s \geq 0$. However, it seems not enough to get the higher regularities of $u$. Fortunately, the local existence result which has been obtained in previous section supplies a unique solution $u$ satisfies

$$
u \in C\left([0, T] ; B^{0, s}\right) \quad \text { and } \quad \nabla_{h}^{\alpha} u \in \widetilde{L}^{2}\left(0, T ; B^{0, s}\right), \quad \partial_{3} u \in \widetilde{L}^{2}\left(0, T ; B^{0, s}\right)
$$

for some positive $T$, which implies $\nabla_{h}^{\alpha} u, \partial_{3} u \in L^{2}\left(0, T ; H^{0, s}\right)$. Thus, there exists a $0<T_{0}<T$ such that $\nabla_{h}^{\alpha} u\left(T_{0}\right), \partial_{3} u\left(T_{0}\right) \in L^{2}\left(\mathbb{R}^{3}\right)$. Without loss of generality, we assume $\nabla_{h}^{\alpha} u_{0}, \partial_{3} u_{0} \in L^{2}\left(\mathbb{R}^{3}\right)$. Using this fact, we have the following lemmas.

Lemma 2. Under the assumptions of Theorem 1, there exists a universal constant $C$ such that for all $t \in\left[0, T^{*}\right)$, the following a priori estimate holds true:

$$
\begin{aligned}
& \left\|\nabla_{h}^{\alpha} u\right\|_{L^{2}}^{2}+\left\|\partial_{3} u\right\|_{L^{2}}^{2} \\
& \quad+\int_{0}^{t}\left\|\partial_{t} u\right\|_{L^{2}}^{2}+\left\|D_{h}^{2 \alpha} u\right\|_{L^{2}}^{2}+\left\|\partial_{3}^{2} u\right\|_{L^{2}}^{2}+\left\|\partial_{3} \nabla_{h}^{\alpha} u\right\|_{L^{2}}^{2} d \tau \\
& \quad \leq C\left(\left\|\nabla_{h}^{\alpha} u_{0}\right\|_{L^{2}}^{2}+\left\|\partial_{3} u_{0}\right\|_{L^{2}}^{2}+t\right) \exp \left\{\int_{0}^{t}\left\|\nabla_{h}^{\alpha} u\right\|_{L^{2}}^{2}+\left\|\partial_{3} u\right\|_{L^{2}}^{2} d \tau\right\}
\end{aligned}
$$

where $T^{*}$ is maximal lifespan of (1.1).

Proof. First, taking the $L^{2}$ scalar product with $\partial_{t} u$ in (1.1), we obtain that

$$
\left\|\partial_{t} u\right\|_{L^{2}}^{2}+\frac{1}{2} \nu_{h} \frac{d}{d t}\left\|D_{h}^{\alpha} u\right\|_{L^{2}}^{2}+\frac{1}{2} \nu_{3} \frac{d}{d t}\left\|\partial_{3} u\right\|_{L^{2}}^{2} \leq \frac{1}{2}\left\|\partial_{t} u\right\|_{L^{2}}^{2}+C\|u \cdot \nabla u\|_{L^{2}}^{2},
$$

that is

$$
\left\|\partial_{t} u^{h}\right\|_{L^{2}}^{2}+\nu_{h} \frac{d}{d t}\left\|D_{h}^{\alpha} u\right\|_{L^{2}}^{2}+\nu_{3} \frac{d}{d t}\left\|\partial_{3} u\right\|_{L^{2}}^{2} \leq C\|u \cdot \nabla u\|_{L^{2}}^{2} .
$$

In order to estimate the convection term, we split $\|u \cdot \nabla u\|_{L^{2}}$ into

$$
\|u \cdot \nabla u\|_{L^{2}} \leq\left\|u^{h} \cdot \nabla_{h} u\right\|_{L^{2}}+\left\|u^{3} \cdot \partial_{3} u\right\|_{L^{2}}:=I+I I .
$$

For $I$, by Hölder inequality, Sobolev embedding theorem and estimate (1.2), one gets

$$
\begin{aligned}
I & \leq\left\|u^{h}\right\|_{L_{h}^{\infty}\left(L_{v}^{4}\right)}\left\|\nabla_{h} u\right\|_{L_{h}^{2}\left(L_{v}^{4}\right)} \\
& \leq C\left\|u^{h}\right\|_{L_{h}^{\infty}\left(L_{v}^{2}\right)}^{\frac{3}{4}}\left\|\partial_{3} u^{h}\right\|_{L_{h}^{\infty}\left(L_{v}^{2}\right)}^{\frac{1}{4}}\left\|\nabla_{h} u\right\|_{L_{h}^{2}\left(L_{v}^{2}\right)}^{\frac{3}{4}}\left\|\partial_{3} \nabla_{h} u\right\|_{L_{h}^{2}\left(L_{v}^{2}\right)}^{\frac{1}{4}} \\
& \leq C\left\|u^{h}\right\|_{L^{2}}^{\frac{3}{2}-\frac{3}{2 \alpha}}\left\|\nabla_{h}^{\alpha} u\right\|_{L^{2}}^{\frac{3}{2 \alpha}}\left\|\partial_{3} u\right\|_{L^{2}}^{\frac{1}{2}-\frac{1}{2 \alpha}}\left\|\partial_{3} \nabla_{h}^{\alpha} u\right\|_{L^{2}}^{\frac{1}{2 \alpha}} .
\end{aligned}
$$

For $I I$, by Hölder inequality and Sobolev embedding theorem, one gets

$$
\begin{aligned}
I I & \leq\left\|u^{3} \partial_{3} u\right\|_{L^{2}\left(\mathbb{R}^{3}\right)} \\
& \leq C\left\|u^{3}\right\|_{L_{h}^{\frac{8}{3}\left(L_{v}^{\infty}\right)}}\left\|\partial_{3} u\right\|_{L_{h}^{8}\left(L_{v}^{2}\right)} \\
& \leq C\left\|u^{3}\right\|_{L_{h}^{\frac{8}{3}}\left(L_{v}^{2}\right)}^{\frac{8}{2}}\left\|\partial_{3} u^{3}\right\|_{L_{h}^{\frac{8}{3}}\left(L_{v}^{2}\right)}^{\frac{1}{2}}\left\|\partial_{3} u\right\|_{L_{h}^{8}\left(L_{v}^{2}\right)} \\
& \leq C\left\|\nabla_{h}^{\frac{1}{4}} u^{3}\right\|_{L^{2}}^{\frac{1}{2}}\left\|\nabla_{h}^{\frac{1}{4}} \partial_{3} u^{3}\right\|_{L^{2}}^{\frac{1}{2}}\left\|\partial_{3} \nabla_{h}^{\frac{3}{4}} u\right\|_{L^{2}} .
\end{aligned}
$$


Using the divergence free condition $\operatorname{div} u=0$ again, one gets

$$
\left\|\nabla_{h}^{\frac{1}{4}} \partial_{3} u^{3}\right\|_{L^{2}}=\left\|\nabla_{h}^{\frac{1}{4}} \operatorname{div}_{h} u^{h}\right\|_{L^{2}} \leq C\|u\|_{L^{2}}^{1-\frac{5}{4 \alpha}}\left\|\nabla_{h}^{\alpha} u\right\|_{L^{2}}^{\frac{5}{4 \alpha}} .
$$

This fact together with (4.4) and basic energy estimate (1.2) yields

$$
I I \leq C\left\|\nabla_{h}^{\alpha} u\right\|_{L^{2}}^{\frac{3}{4 \alpha}}\left\|\partial_{3} u\right\|_{L^{2}}^{1-\frac{3}{4 \alpha}}\left\|\partial_{3} \nabla_{h}^{\alpha} u\right\|_{L^{2}}^{\frac{3}{4 \alpha}} .
$$

Next, we consider the following Stokes system

$$
\left\{\begin{array}{l}
\nu_{h} D_{h}^{2 \alpha} u-\nu_{3} \partial_{3}^{2} u+\nabla \Pi=-\partial_{t} u-u \cdot \nabla u \\
\operatorname{div} u=0
\end{array}\right.
$$

Taking $L^{2}$ estimate of elliptic equation yields

$$
\begin{aligned}
& \nu_{h}\left\|D_{h}^{2 \alpha} u\right\|_{L^{2}}^{2}+\nu_{3}\left\|\partial_{3}^{2} u\right\|_{L^{2}}^{2}+\left(\nu_{h}+\nu_{3}\right)\left\|\partial_{3} \nabla_{h}^{\alpha} u\right\|_{L^{2}}^{2}+\|\nabla \Pi\|_{L^{2}}^{2} \\
& \quad \leq C_{1}\left(\left\|\partial_{t} u\right\|_{L^{2}}^{2}+\|u \cdot \nabla u\|_{L^{2}}^{2}\right) .
\end{aligned}
$$

Making use of (4.3)-(4.4) in (4.5), we have

$$
\begin{aligned}
& \nu_{h}\left\|D_{h}^{2 \alpha} u\right\|_{L^{2}}^{2}+\nu_{3}\left\|\partial_{3}^{2} u\right\|_{L^{2}}^{2}+\left(\nu_{h}+\nu_{3}\right)\left\|\partial_{3} \nabla_{h}^{\alpha} u\right\|_{L^{2}}^{2}+\|\nabla \Pi\|_{L^{2}}^{2} \\
& \quad \leq C_{1}\left(\left\|\partial_{t} u\right\|_{L^{2}}^{2}+\left\|\nabla_{h}^{\alpha} u\right\|_{L^{2}}^{\frac{6}{2 \alpha-1}}\left\|\partial_{3} u\right\|_{L^{2}}^{\frac{2 \alpha-2}{2 \alpha-1}}+\left\|\partial_{3} u\right\|_{L^{2}}^{2}\left\|\nabla_{h}^{\alpha} u\right\|_{L^{2}}^{\frac{6}{4 \alpha-3}}\right) .
\end{aligned}
$$

Hence, combining (4.6) with (4.2), applying Young's inequality implies

$$
\begin{aligned}
& \frac{d}{d t}\left(\left\|D_{h}^{\alpha} u\right\|_{L^{2}}^{2}+\left\|\partial_{3} u\right\|_{L^{2}}^{2}\right)+\left\|\partial_{t} u\right\|_{L^{2}}^{2}+\left\|D_{h}^{2 \alpha} u\right\|_{L^{2}}^{2}+\left\|\partial_{3}^{2} u\right\|_{L^{2}}^{2}+\left\|\partial_{3} \nabla_{h}^{\alpha} u\right\|_{L^{2}}^{2} \\
& \quad \leq C\left\|\nabla_{h}^{\alpha} u\right\|_{L^{2}}^{\frac{6}{2 \alpha-1}}\left\|\partial_{3} u\right\|_{L^{2}}^{\frac{2 \alpha-2}{2 \alpha-1}}+C\left\|\partial_{3} u\right\|_{L^{2}}^{2}\left\|\nabla_{h}^{\alpha} u\right\|_{L^{2}}^{\frac{6}{4 \alpha-3}} .
\end{aligned}
$$

Performing a time integration on $[0, t]$ yields

$$
\begin{aligned}
& \left\|\nabla_{h}^{\alpha} u\right\|_{L^{2}}^{2}+\left\|\partial_{3} u\right\|_{L^{2}}^{2}+\int_{0}^{t}\left(\left\|\partial_{t} u\right\|_{L^{2}}^{2}+\left\|D_{h}^{2 \alpha} u\right\|_{L^{2}}^{2}+\left\|\partial_{3}^{2} u\right\|_{L^{2}}^{2}+\left\|\partial_{3} \nabla_{h}^{\alpha} u\right\|_{L^{2}}^{2}\right) d \tau \\
& \leq C\left\|\nabla_{h}^{\alpha} u_{0}\right\|_{L^{2}}^{2}+C\left\|\partial_{3} u_{0}\right\|_{L^{2}}^{2}+C \int_{0}^{t}\left\|\nabla_{h}^{\alpha} u\right\|_{L^{2}}^{\frac{4}{\alpha}} d \tau \\
& \quad+C \int_{0}^{t}\left(\left\|\nabla_{h}^{\alpha} u\right\|_{L^{2}}^{\frac{6}{2 \alpha-1}}\left\|\partial_{3} u\right\|_{L^{2}}^{\frac{2 \alpha-2}{2 \alpha-1}}+\left\|\partial_{3} u\right\|_{L^{2}}^{2}\left\|\nabla_{h}^{\alpha} u\right\|_{L^{2}}^{\frac{6}{4 \alpha-3}}\right) d \tau
\end{aligned}
$$

Since $\alpha \geq \frac{3}{2}$, according to Young's inequality, one can deduce that

$$
\begin{gathered}
\int_{0}^{t}\left\|\nabla_{h}^{\alpha} u\right\|_{L^{2}}^{\frac{6}{2 \alpha-1}}\left\|\partial_{3} u\right\|_{L^{2}}^{\frac{2 \alpha-2}{2 \alpha-1}} d \tau \leq C \int_{0}^{t}\left(\left\|\nabla_{h}^{\alpha} u\right\|_{L^{2}}^{4}+\left\|\partial_{3} u\right\|_{L^{2}}^{4}+1^{\frac{8 \alpha-4}{6 \alpha-8}}\right) d \tau \\
\int_{0}^{t}\left\|\partial_{3} u\right\|_{L^{2}}^{2}\left\|\nabla_{h}^{\alpha} u\right\|_{L^{2}}^{\frac{6}{4 \alpha-3}} d \tau \leq C \int_{0}^{t}\left(\left\|\nabla_{h}^{\alpha} u\right\|_{L^{2}}^{4}+\left\|\partial_{3} u\right\|_{L^{2}}^{4}+1^{\frac{2 \alpha-\frac{3}{2}}{2 \alpha-3}}\right) d \tau
\end{gathered}
$$

Finally, we obtain that

$$
\begin{aligned}
& \left\|\nabla_{h}^{\alpha} u\right\|_{L^{2}}^{2}+\left\|\partial_{3} u\right\|_{L^{2}}^{2}+\int_{0}^{t}\left(\left\|\partial_{t} u\right\|_{L^{2}}^{2}+\left\|D_{h}^{2 \alpha} u\right\|_{L^{2}}^{2}+\left\|\partial_{3}^{2} u\right\|_{L^{2}}^{2}+\left\|\partial_{3} \nabla_{h}^{\alpha} u\right\|_{L^{2}}^{2}\right) d \tau \\
& \leq C\left(\left\|\nabla_{h}^{\alpha} u_{0}\right\|_{L^{2}}^{2}+\left\|\partial_{3} u_{0}\right\|_{L^{2}}^{2}+t\right)+C \int_{0}^{t}\left(\left\|\nabla_{h}^{\alpha} u\right\|_{L^{2}}^{4}+\left\|\partial_{3} u\right\|^{4}\right) d \tau
\end{aligned}
$$

Then Gronwall's inequality completes the proof of this lemma. 
Remark 1. Combining Lemma 2 with (1.2), we find that

$$
\int_{0}^{t}\left\|\nabla_{h}^{\alpha} u\right\|_{L^{2}}^{2}+\left\|\partial_{3} u\right\|_{L^{2}}^{2} d \tau \leq C
$$

for all $t \in\left[0, T^{*}\right)$. This means the right hand side of (4.1) is finite.

Completion of the proof of the Theorem 1.1. First, we treat on the case that $\nabla_{h}^{\alpha} u_{0} \in L^{2}\left(\mathbb{R}^{3}\right)$ and $\partial_{3} u_{0} \in L^{2}\left(\mathbb{R}^{3}\right)$. For $\alpha \geq \frac{3}{2}$, Lemma 2 implies that $\nabla_{h}^{2 \alpha} u, \partial_{3}^{2} u, \partial_{3} \nabla_{h}^{\alpha} u$ belong to $L^{2}\left(0, T^{*} ; L^{2}\right)$. If $T^{*}<+\infty$, by Hölder inequality, we have

$$
\begin{aligned}
& \int_{0}^{t}\|u(\tau)\|_{L_{v}^{\infty}\left(L_{h}^{2}\right)}^{\frac{4 \alpha-2}{4 \alpha-3}}\left\|\nabla_{h}^{\alpha} u(\tau)\right\|_{L_{v}^{\infty}\left(L_{h}^{2}\right)}^{\frac{2}{4 \alpha-3}} d \tau \\
& \quad \leq C \int_{0}^{t}\|u\|_{L^{2}}^{\frac{2 \alpha-1}{4 \alpha-3}}\left\|\partial_{3} u\right\|_{L^{2}}^{\frac{2 \alpha-1}{4 \alpha-3}}\left\|\partial_{3} \nabla_{h}^{\alpha} u\right\|_{L^{2}}^{\frac{1}{4 \alpha-3}}\left\|\nabla_{h}^{\alpha} u\right\|_{L^{2}}^{\frac{1}{4 \alpha-3}} d \tau \\
& \quad \leq C\|u\|_{L_{t}^{\infty}\left(L^{2}\right)}^{\frac{2 \alpha-1}{4 \alpha-3}}\left\|\partial_{3} u\right\|_{L_{t}^{\infty}\left(L^{2}\right)}^{\frac{2 \alpha-1}{4 \alpha-3}}\left\|\nabla_{h}^{\alpha} u\right\|_{L_{t}^{\infty}\left(L^{2}\right)}^{\frac{1}{4 \alpha-3}}\left\|\partial_{3} \nabla_{h}^{\alpha} u\right\|_{L_{t}^{2}\left(L^{2}\right)}^{\frac{1}{4 \alpha-3}} \leq C
\end{aligned}
$$

for all $t \in\left[0, T^{*}\right)$. Similarly,

$$
\int_{0}^{t}\|u(\tau)\|_{L_{v}^{\infty}\left(L_{h}^{2}\right)}^{\frac{4 \alpha-2}{4 \alpha-1}}\left\|\nabla_{h}^{\alpha} u(\tau)\right\|_{L_{v}^{\infty}\left(L_{h}^{2}\right)}^{\frac{2}{4 \alpha-1}} d \tau \leq C
$$

and

$$
\int_{0}^{t}\|u(\tau)\|_{L_{v}^{\infty}\left(L_{h}^{2}\right)}^{\frac{2 \alpha-2}{2 \alpha-1}}\left\|\nabla_{h}^{\alpha} u(\tau)\right\|_{L_{v}^{\infty}\left(L_{h}^{2}\right)}^{\frac{2}{2 \alpha-1}} d \tau \leq C
$$

for all $t \in\left[0, T^{*}\right)$. Hence the blow up criterion satisfied, which implies $T^{*}=$ $+\infty$.

Next, when $u_{0} \in B^{0, s}\left(\mathbb{R}^{3}\right)$, Theorem 2 supplies a unique solution $u$ with

$$
u \in C\left(\left[0, T^{*}\right), B^{0, s}\right) \quad \text { and } \quad \nabla_{h}^{\alpha} u \in \widetilde{L}^{2}\left(0, T^{*} ; B^{0, s}\right), \quad \partial_{3} u \in \widetilde{L}^{2}\left(0, T^{*} ; B^{0, s}\right) .
$$

Hence there exists a $T_{0}<T^{*}$ such that $\nabla_{h}^{\alpha} u\left(T_{0}\right), \partial_{3} u\left(T_{0}\right)$ belong to $L^{2}\left(\mathbb{R}^{3}\right)$. In the previous case we get a unique solution $\widetilde{u}$ on $\left[T_{0}, \infty\right)$, with data $u\left(T_{0}\right)$. Uniqueness ensures that $\widetilde{u} \equiv u$ on $\left[T_{0}, T^{*}\right)$. Therefore $u$ can be continued globally.

\section{Acknowledgement}

This work is supported partially by NSFC 11271322 and 11331005.

\section{References}

[1] H. Bahouri, J.Y. Chemin and R. Danchin. Fourier Analysis and Nonlinear Partial Differential Equations. Grundlehren Math. Wiss. Springer, 2011.

[2] J.M. Bony. Calcul symbolique et propagation des singularités pour équations aux dérivées partielles nonlinéaires. Ann. Sci. École Norm. Sup., 14:209-246, 1981. 
[3] J.Y. Chemin, B. Desjardins, I. Gallagher and E. Grenier. Anisotropy and dispersion in rotating fluids. Stud. Math. Appl., 31:171-192, 2002. http://dx.doi.org/10.1016/S0168-2024(02)80010-8.

[4] J.Y. Chemin and N. Lerner. Flot de champs de vecteurs non Lipschitziens et équations de Navier-Stokes. J. Differential Equations, 121:314-228, 1995. http://dx.doi.org/10.1006/jdeq.1995.1131.

[5] D. Fang and Rui. Z. Zi. On the well-posedness of inhomogeneous hyperdissipative Navier-Stokes equations. Discrete Contin. Dyn. Syst., 33:3517-3541, 2013. http://dx.doi.org/10.3934/dcds.2013.33.3517.

[6] D. Iftimie. The resolution of the Navier-Stokes equations in anisotropic spaces. Rev. Mat. Iberoamericana, 15:1-36, 1999. http://dx.doi.org/10.4171/RMI/248.

[7] N. Katz and N. Pavlović. A cheap Caffarelli-Kohn-Nirenberg inequality for the Navier-Stokes equaiton with hyper-dissipation. Geom. Funct. Anal., 12:355-379, 2002. http://dx.doi.org/10.1007/s00039-002-8250-z.

[8] M. Majdoub and M. Paicu. Uniform local existence for inhomogeneous rotating fluid equations. J. Dynam. Differential Equations, 21:21-44, 2009. http://dx.doi.org/10.1007/s10884-008-9120-7.

[9] M. Paicu. Equation periodique de Navier-Stokes sans viscosite dans une direction. Comm. Partial Differential Equations, 30:1107-1140, 2005. http://dx.doi.org/10.1080/036053005002575529.

[10] T. Tao. Global regularity for a logarithmically supercritical hyperdissipative Navier-Stokes equation. Anal. PDE, 22:361-366, 2009. http://dx.doi.org/10.2140/apde.2009.2.361.

[11] T. Zhang. Global wellposed problem for the 3-d incompressible anisotropic Navier-Stokes equations in an anisotropic space. Commun. Math. Phys., 287:211-224, 2009. http://dx.doi.org/10.1007/s00220-008-0631-1. 